\title{
Numerical simulation of surface acoustic wave actuated cell sorting
}

\author{
Thomas Franke ${ }^{1 *}$, Ronald H.W. Hoppe ${ }^{2,3 \dagger}$, Christopher Linsenmann ${ }^{3 \neq}$, Kidist Zeleke ${ }^{2 \S}$
}

1 Institute of Physics, University of Augsburg, Universitätstr. 1, 86159 Augsburg, Germany

2 Department of Mathematics, University of Houston, 669 P.G. Hoffman, Houston, TX 77204-3008, USA

3 Institute of Mathematics, University of Augsburg, Universitätsstr. 14, 86159 Augsburg, Germany

Received 29 February 2012; accepted 28 September 2012

\begin{abstract}
We consider the mathematical modeling and numerical simulation of high throughput sorting of two different types of biological cells (type I and type II) by a biomedical micro-electro-mechanical system (BioMEMS) whose operating behavior relies on surface acoustic wave (SAW) manipulated fluid flow in a microchannel. The BioMEMS consists of a separation channel with three inflow channels for injection of the carrier fluid and the cells, two outflow channels for separation, and an interdigital transducer (IDT) close to the lateral wall of the separation channel for generation of the SAWs. The cells can be distinguished by fluorescence. The inflow velocities are tuned so that without SAW actuation a cell of type I leaves the device through a designated outflow channel. However, if a cell of type II is detected, the IDT is switched on and the SAWs modify the fluid flow so that the cell leaves the separation channel through the other outflow boundary. The motion of a cell in the carrier fluid is modeled by the Finite Element Immersed Boundary method (FE-IB). Numerical results are presented that illustrate the feasibility of the surface acoustic wave actuated cell sorting approach.
\end{abstract}

MSC: $\quad 65 \mathrm{M} 60,74 \mathrm{~L} 15,76 Z 05,92 \mathrm{C} 10,92 \mathrm{C} 50$

Keywords: Surface acoustic wave actuated cell sorting • Biomedical micro-electro-mechanical system • Finite element immersed boundary method

(c) Versita Sp. z o.o. 


\section{Introduction}

Biomedical Micro-Electro-Mechanical Systems (BioMEMS) are miniaturized laboratories on a chip (lab-on-a-chip) that can be used for various biomedical and biochemical purposes such as hybridization in genomics, protein profiling in proteomics, and cytometry in cell analysis. In this project, we will focus on high throughput cell sorting in microfluidic channels which has significant applications in basic cell biology, cancer research, clinical diagnostics, drug design in pharmacology, tissue engineering in reproductive medicine, and transplantation immunology $[8,10,16,25,29]$.

The working horses in cell sorting are still centrifugal methods where a sample is spun in a rotating chamber such that heavier cells are separated from lighter ones $[19,24]$. Other bioengineering technologies are based on electrokinetic/dielectrophoretic methods where an external electric field is used to separate cells with different charge or polarization properties $[9,31,33]$, or on magnetic methods, where cells (particles) of interest are labeled with magnetic materials and separated from the rest by applying an external magnetic field [23, 32]. Acoustic techniques rely on bulk acoustic wave (BAW)-based acoustophoresis [22] or standing surface acoustic waves (SSAW) [26, 27] to separate cells (particles) of different densities or sizes.

All the methodologies mentioned before heavily rely on different properties of the cells or particles in the sample (density/size, charge/polarization, magnetic labeling) and hence are restricted to specific applications. A very recent technology, which does not depend on such contrasts, is surface acoustic wave actuated cell sorting (SAWACS) [12, 13]. It combines the advantages of SAW generated acoustic streaming in microfluidic polydimethylsiloxane (PDMS) devices and fluorescence activated cell sorting (FACS).

The separation channel is placed on top of a plastic chip partially coated by a piezoelectric substrate to enable the generation and propagation of surface acoustic waves (SAWs). The channel has length of $350 \mu m$, a width of $220 \mu m$, and a height of $50 \mu \mathrm{m}$. It features an injection channel at the center of the left boundary (highlighted in green in Figure 1), two adjacent inflow channels, and two outflow channels at the right boundary. An interdigital transducer (IDT) is placed close to the lower lateral wall. Once a cell has entered the separation channel and is recognized as a cell designated to leave the upper outlet, the IDT is switched on and generates the SAWs. The induced acoustic streaming deflects the focussing stream towards the upper outlet, cf. Figure 1 (bottom). Without SAW actuation, the velocities at the inflow channels and the injection channel are tuned in such a way that the cell leaves the main channel through the lower outlet, cf. Figure 1 (top). The device has been successfully tested for cell sorting involving different cell types such as human keratinocytes, murine fibroblasts cells, and melanoma cells [13]. In this paper, we will be concerned with SAW actuated cell sorting both for viscoelastic cells such as red blood cells (RBCs) and malignant breast cancer cells.

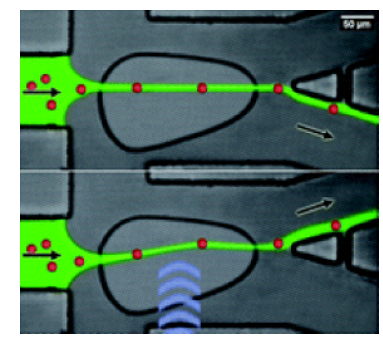

Figure 1. Surface acoustic wave actuated cell sorting (SAWACS) in a microfluidic PDMS channel (prepared in the lab of the first author).

\section{The mathematical model equations}

We use standard notation from Lebesgue and Sobolev space theory, cf., e.g., [30]. In particular, for a bounded domain $\Omega \subset \mathbb{R}^{d}, d \in \mathbb{N}$, we denote by $L^{2}(\Omega)$ and $L^{2}(\Omega)=L^{2}(\Omega)^{d}$ the Hilbert space of square integrable scalar- and vectorvalued functions on $\Omega$, equipped with the inner product $(\cdot, \cdot)_{0, \Omega}$ and the associated norm $\|\cdot\|_{0, \Omega}$, respectively. Further, we denote by $H^{s}(\Omega), s \in \mathbb{R}_{+}$, the Sobolev space of vector-valued functions with the inner product $(\cdot, \cdot)_{s, \Omega}$ and the associated 
norm $\|\cdot\|_{s, \Omega}$. The space $\mathrm{H}_{0, \Gamma^{\prime}}^{s}(\Omega)$ is the subspace with vanishing trace on $\Gamma^{\prime} \subseteq \Gamma$. We will omit the subindex $\Gamma^{\prime}$ if $\Gamma^{\prime}=\Gamma$. $\mathrm{H}^{-s}(\Omega)$ stands for the dual space of $\mathrm{H}_{0}^{s}(\Omega)$ with $\langle\cdot, \cdot\rangle$ referring to the dual product. The space $\mathbf{H}^{s}(\bar{\Omega}) \subset \mathrm{H}^{s}(\Omega)$ is the subspace of all $\left.\mathbf{u}\right|_{\Omega}$ where $\mathbf{u} \in \mathrm{H}^{s}\left(\mathbb{R}^{d}\right)$ and $\left\langle\left.\mathbf{u}\right|_{\Omega}, \boldsymbol{\varphi}\right\rangle=\langle\mathbf{u}, \tilde{\varphi}\rangle$ for all $\boldsymbol{\varphi} \in \mathrm{C}_{0}^{\infty}(\Omega)$ with $\tilde{\varphi}$ referring to the continuation of $\boldsymbol{\varphi}$ by zero outside $\Omega$. We denote by $\mathrm{H}^{s-1 / 2}\left(\Gamma^{\prime}\right), s \geq 1$, the trace space of vector-valued functions on $\Gamma^{\prime}$. We further refer to $\mathbf{H}_{00}^{s-1 / 2}\left(\Gamma^{\prime}\right)$ as the space of functions whose extensions by zero to $\Gamma \backslash \Gamma^{\prime}$ belong to $\mathbf{H}^{s-1 / 2}(\Gamma)$. Finally, we denote by $C^{k, \mu}(\Omega)$ and $C^{k, \mu}(\Omega), k \in \mathbb{N}_{0}, \mu \in(0,1 / 2)$, the Banach spaces of $k$-times continuously differentiable scalar- and vector-valued functions on $\Omega$ whose derivatives of order $k$ are Hölder continuous of order $\mu$.

Moreover, for $T>0$ and a Banach space $Z(Z)$ of scalar (vector-valued) functions, we denote by $L^{2}((0, T), Z)\left(\mathrm{L}^{2}((0, T), \mathbf{Z})\right)$ the Hilbert space and by $C([0, T], Z)(C([0, T], Z))$ the Banach space of functions $v:[0, T] \rightarrow Z(\mathbf{v}:[0, T] \rightarrow Z)$ with norms

$$
\|v\|_{L^{2}((0, T), Z)}=\left(\int_{0}^{T}\|v(t)\|_{Z}^{2} d t\right)^{1 / 2}, \quad\|v\|_{C([0, T], Z)}=\max _{t \in[0, T]}\|v(t)\|_{Z},
$$

and analogous settings in the vector-valued case. The spaces $H^{s}((0, T), Z), s \in \mathbb{R}_{+},\left(\mathbf{H}^{s}((0, T), \mathbf{Z})\right)$ are defined likewise.

\subsection{Incompressible Navier-Stokes equations}

We consider a microchannel $\Omega=\left(0, a_{1}\right) \times\left(0, a_{2}\right), a_{i}>0$, with three inflow boundaries $\Gamma_{\text {in }}^{(i)}=\{0\} \times\left(b_{1}^{(i)}, b_{2}^{(i)}\right), 0<b_{1}^{(i)}<$ $b_{2}^{(i)}, 1 \leq i \leq 3$, two outflow boundaries $\Gamma_{\text {out }}^{(i)}=\left\{a_{1}\right\} \times\left(c_{1}^{i)}, c_{2}^{(i)}\right), 0<c_{1}^{(i)}<c_{2}^{(i)}<a_{2}, 1 \leq i \leq 2$, and a boundary $\Gamma_{\mathrm{ac}}=\left(a_{1} / 2-d_{1}, a_{1} / 2+d_{1}\right) \times\{0\}$, where the SAWs enter the channel. We set $\Gamma_{D}=\partial \Omega \backslash\left(\bigcup_{i=1}^{3} \bar{\Gamma}_{\text {in }}^{(i)} \cup \bigcup_{i=1}^{2} \bar{\Gamma}_{\text {out }}^{(i)} \cup \bar{\Gamma}_{\mathrm{ac}}\right)$ and $\Gamma^{\prime}=\bigcup_{i=1}^{3} \Gamma_{\text {in }}^{(i)} \cup \Gamma_{\text {ac }} \cup \Gamma_{\mathrm{D}}$, cf. Figure 2. For $T>0$, we further set $Q=\Omega \times(0, T], \Sigma_{\text {in }}^{(i)}=\Gamma_{\text {in }}^{(i)} \times(0, T], 1 \leq i \leq 3$, $\Sigma_{\text {out }}^{(i)}=\Gamma_{\text {out }}^{(i)} \times(0, T], 1 \leq i \leq 2, \Sigma_{\mathrm{ac}}=\Gamma_{\mathrm{ac}} \times(0, T]$, and $\Sigma_{\mathrm{D}}=\Gamma_{\mathrm{D}} \times(0, T]$.

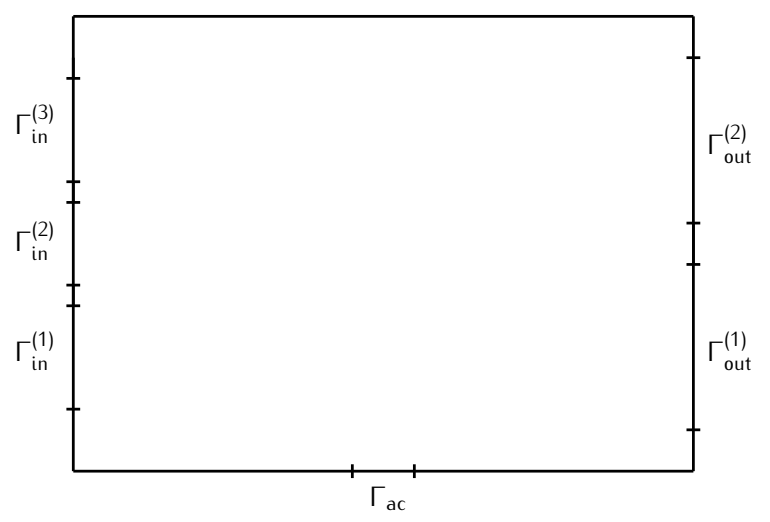

Figure 2. Microchannel with three inflow boundaries $\Gamma_{\text {in }}^{(i)}, 1 \leq i \leq 3$, two outflow boundaries $\Gamma_{\text {out }}^{(i)}, 1 \leq i \leq 2$, and a boundary $\Gamma_{\text {ac }}$ where the SAWs enter the channel.

We denote by $\mathrm{v}$ and $p$ the velocity field and the pressure and refer to $\sigma(\mathrm{v}, p)=\eta \mathrm{D}(\mathrm{v})-p \mathrm{I}$ as the stress tensor, where $\eta$ is the dynamic viscosity of the carrier fluid and $\mathrm{D}(\mathrm{v})=\left(\nabla \mathrm{v}+(\nabla \mathrm{v})^{T}\right) / 2$ stands for the rate of deformation tensor. We impose inflow velocities $v_{i n}^{(i)}$ at the inflow boundaries $\Sigma_{\text {in }}^{(i)}, 1 \leq i \leq 3$, do-nothing boundary conditions at the outflow boundaries $\Sigma_{\text {out }}^{(i)}, 1 \leq i \leq 2$, and zero velocity at $\Sigma_{\mathrm{D}}$. Moreover, we have $\mathbf{v}=\mathbf{v}_{\mathrm{ac}}$ at $\Sigma_{\mathrm{ac}}$ with $\mathbf{v}_{\mathrm{ac}}=\mathbf{0}$ in case of no SAW actuation and $\mathbf{v}_{\mathrm{ac}}=\partial \mathbf{u} / \partial t$ in case the IDT is switched on. Here, $\mathbf{u}$ stands for the displacement vector of the SAW which will be specified in the following subsection 2.2. Finally, we denote by $v^{(0)}$ the initial velocity and by $F$ a force density which reflects the impact of the immersed cell on the carrier fluid and which will be derived in subsection 2.3 . 
The initial-boundary value problem for the incompressible Navier-Stokes equations then reads as follows:

$$
\begin{aligned}
\rho \frac{\partial \mathbf{v}}{\partial t}+\rho(\mathbf{v} \cdot \nabla) \mathbf{v}-\nabla \cdot \boldsymbol{\sigma}(\mathbf{v}, p)=\mathrm{F} & \text { in } \quad, \\
\nabla \cdot \mathbf{v}=0 & \text { in } Q, \\
\mathbf{v}=\mathbf{v}_{\text {in }}^{(i)} & \text { on } \Sigma_{\text {in }}^{(i)} \quad 1 \leq i \leq 3, \\
\boldsymbol{\sigma}(\mathbf{v}, p) \mathbf{n}=\mathbf{0} & \text { on } \Sigma_{\text {out }}^{(i)}, \\
\mathbf{v}=\mathbf{v}_{\mathrm{ac}} & \text { on } \Sigma_{\mathrm{ac}} \\
\mathbf{v}=\mathbf{0} & \text { on } \Sigma_{\mathrm{D}}, \\
\mathbf{v}=\mathbf{v}^{(0)} & \text { in } \Omega .
\end{aligned}
$$

Introducing the function spaces

$$
\begin{aligned}
\mathrm{V}(0, T) & =\mathrm{H}^{1}\left((0, T), \mathrm{H}^{-1}(\Omega)\right) \cap \mathrm{L}^{2}\left((0, T), \mathrm{H}^{1}(\Omega)\right), \\
\mathrm{W}(0, T) & \left.=\{\mathrm{v} \in \mathrm{V}(0, T): \mathrm{v}\rceil_{\Sigma_{\mathrm{in}}^{(i)}}=\mathrm{v}_{\mathrm{in}}^{(i)}, 1 \leq i \leq 3,\left.\mathrm{v}\right|_{\Sigma_{\mathrm{ac}}}=\mathrm{v}_{\mathrm{ac}},\left.\mathrm{v}\right|_{\Sigma_{\mathrm{D}}}=0\right\}, \\
Q(0, T) & =L^{2}\left((0, T), L^{2}(\Omega)\right),
\end{aligned}
$$

the weak formulation of the Navier-Stokes equations requires the computation of $(\mathbf{v}, p) \in \mathbf{W}(0, T) \times Q(0, T)$ such that for all $\mathbf{w} \in \mathrm{H}_{0, \Gamma^{\prime}}^{1}(\Omega)$ and all $q \in L^{2}(\Omega)$ there holds

$$
\begin{aligned}
\left\langle\rho \frac{\partial \mathbf{v}}{\partial t}, \mathbf{w}\right\rangle_{\mathrm{H}^{-1}, \mathrm{H}^{1}}+a(\mathbf{v}, \mathbf{w})-b(p, \mathbf{w}) & =\ell(\mathbf{w}) \\
b(q, \mathbf{v}) & =0 \\
\mathrm{v}(\cdot, 0) & =\mathbf{v}^{(0)}
\end{aligned}
$$

Here, $a(\cdot, \cdot), b(\cdot, \cdot)$, and the functional $\ell(\cdot)$ are given by

$$
a(\mathbf{v}, \mathbf{w})=(\rho(\mathbf{v} \cdot \nabla) \mathbf{v}, \mathbf{w})_{0, \Omega}+(\eta \nabla \mathbf{v}, \nabla \mathbf{w})_{0, \Omega}, \quad b(p, \mathbf{v})=(p, \nabla \cdot \mathbf{v})_{0, \Omega}, \quad \ell(\mathbf{w})=\langle\mathbf{F}, \mathbf{w}\rangle_{\mathrm{H}^{-1}, \mathbf{H}_{0}^{1}}
$$

\subsection{Surface acoustic wave actuation}

In SAW actuated cell sorting, the separation channel is placed on top of a plastic chip partially coated by a piezoelectric substrate such as lithium niobate $\left(\mathrm{LiNbO}_{3}\right)$. The $\mathrm{SAWs}$ are generated by an interdigital transducer (IDT) close to the wall of the channel with its aperture pointing towards the wall. The IDT features fingers substantially parallel to one another. A static electric field $\mathrm{E}$ is applied to generate a strain which varies across the aperture of the IDT. The electric field is either perpendicular or parallel to the fingers and created by applying an AC voltage between two correspondingly positioned conductors. The piezoelectric effect thus leads to SAWs that travel in the direction of the wall, enter the fluid filled microchannel, and thus manipulate the flow field in the channel.

In piezoelectric materials, the stress tensor $\boldsymbol{\sigma}$ depends linearly on the electric field $E$ according to the generalized Hooke's law

$$
\sigma(\mathrm{u}, \mathrm{E})=\mathbf{c} \varepsilon(\mathrm{u})-\mathrm{eE},
$$

where $\varepsilon(\mathbf{u})=\left(\nabla \mathbf{u}+(\nabla \mathbf{u})^{T}\right) / 2$ is the linearized strain tensor and $\mathbf{u}$ denotes the mechanical displacement. Moreover, $\mathbf{c}$ and e refer to the symmetric fourth order elasticity tensor and the symmetric third order piezoelectric tensor, respectively. Hence, the application of an electric field causes a displacement of the material. The origin of the piezoelectric effect is related to an asymmetry in the unit cell of a piezoelectric crystal and can be observed only in materials with a polar axis, cf., e.g., $[11,18]$. 


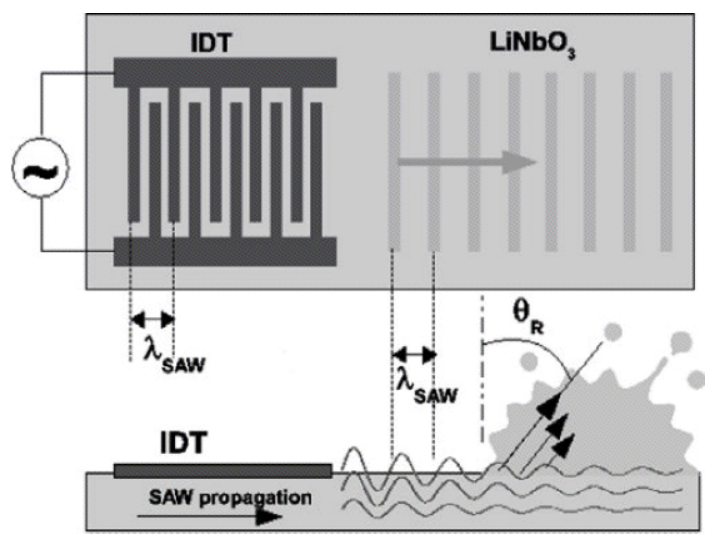

Figure 3. Interdigital Transducer IDT (top) and motion of the SAW in the sagittal plane (right).

Since the frequency of the electromagnetic wave is small compared to the frequency of the generated acoustic wave, a coupling will be neglected. Further, the electric field will be considered as quasistatic and irrotational so that it can be expressed as the gradient of an electric potential $\Phi$, i.e., $E=-\nabla \Phi$. Moreover, piezoelectric materials are nearly perfect insulators and hence, the only remaining quantity of interest in Maxwell's equations is the dielectric displacement D which is related to the electric field by the constitutive equation

$$
\mathrm{D}=\epsilon \mathrm{E}+\mathrm{P}
$$

where $\boldsymbol{\epsilon}$ is the electric permittivity of the material and $\mathbf{P}$ stands for the polarization which depends linearly on the displacement $\mathbf{u}$ according to $\mathbf{P}=\mathbf{e} \varepsilon(\mathbf{u})$. We assume that the piezoelectric material with density $\rho$ occupies some domain $\Omega_{1}$ with boundary $\Gamma_{1}=\partial \Omega_{1}$ and exterior unit normal $\mathbf{n}_{1}$ such that

$$
\begin{array}{ll}
\Gamma_{1}=\bar{\Gamma}_{E, D} \cup \bar{\Gamma}_{E, N}, & \Gamma_{E, D} \cap \Gamma_{E, N}=\emptyset, \\
\Gamma_{1}=\bar{\Gamma}_{p, D} \cup \bar{\Gamma}_{p, N}, & \Gamma_{p, D} \cap \Gamma_{p, N}=\emptyset,
\end{array}
$$

where $\Gamma_{E, D}$ is a rectangular subdomain of the upper boundary of $\Gamma_{1}$ and $\Gamma_{E, N}=\Gamma_{1} \backslash \Gamma_{E, D}$. Given boundary data $\Phi_{E, D}$ on $\Gamma_{E, D}$, the pair $(\mathbf{u}, \Phi)$ satisfies the following initial-boundary value problem for the piezoelectric equations, cf. [15],

$$
\begin{array}{rc}
\rho_{p} \frac{\partial^{2} \mathbf{u}}{\partial t^{2}}-\nabla \cdot \boldsymbol{\sigma}(\mathbf{u}, \mathrm{E})=0 & \text { in } Q_{1}=\Omega_{1} \times\left(0, T_{1}\right), \\
\nabla \cdot \mathbf{D}(\mathbf{u}, \mathrm{E})=0 & \text { in } Q_{1}, \\
\mathbf{u}=0 \quad \text { on } \Gamma_{p, D}, & \mathbf{n}_{1} \cdot \boldsymbol{\sigma}=\boldsymbol{\sigma}_{\mathbf{n}_{1}} \quad \text { on } \Gamma_{p, N}, \\
\Phi=\Phi_{E, D} \quad \text { on } \Gamma_{E, D}, & \mathbf{n}_{1} \cdot \mathbf{D}=D_{\mathbf{n}_{1}} \quad \text { on } \Gamma_{E, N}, \\
\mathbf{u}(\cdot, 0)=0, \quad \frac{\partial \mathbf{u}}{\partial t}(\cdot, 0)=0 \quad \text { in } \Omega_{1} .
\end{array}
$$

For an ideal piezoelectric material, the SAW behaves like a Rayleigh wave which propagates in the sagittal plane spanned by the unit surface normal and the real wave vector $\mathbf{k}$, i.e., the $\left(x_{1}, x_{3}\right)$-plane with respect to the coordinate system in Figure 4. SAWs are strictly confined to the limiting surface of the piezoelectric substrate and practically nil outside a relatively narrow zone. To be precise, the amplitude of the displacement u decays exponentially with depth into the substrate [18]. In true Rayleigh waves, most of the energy $(90 \%)$ is concentrated within one wavelength from the surface. The mechanical displacement $\mathbf{u}$ should thus vanish as $x_{3} \rightarrow \infty$, and since $x_{1}$ is the direction of propagation of the wave, the dependence of $\mathbf{u}$ on the $x_{2}$ coordinate can be neglected. Moreover, if the harmonically excited IDT is 

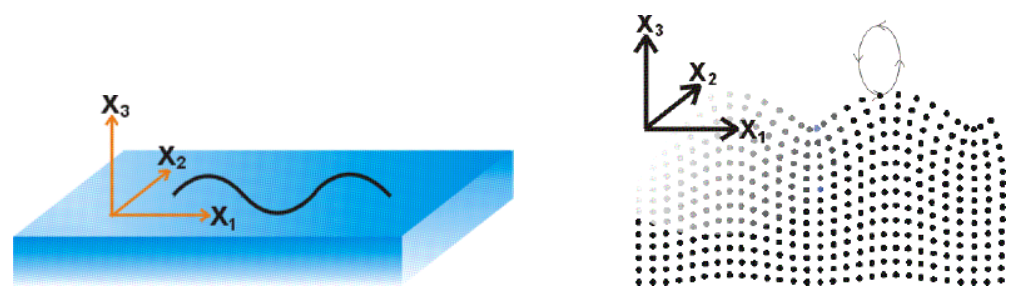

Figure 4. Surface acoustic waves: coordinate system (left) and Rayleigh wave (right).

placed close to the lateral wall of the microchannel and we restrict ourselves to a two-dimensional scenario, we may assume $\mathbf{u}=\left(u_{1}, u_{2}\right)$ with

$$
u_{1}=0, \quad u_{2}=a \sin (2 \pi f t) \quad \text { on } \quad \Sigma_{\mathrm{ac}},
$$

where $a$ is the amplitude and $f$ stands for the operating frequency of the IDT. This leads to $v_{\mathrm{ac}}=\left(v_{\mathrm{ac}, 1}, v_{\mathrm{ac}, 2}\right)^{T}$ with

$$
v_{\mathrm{ac}, 1}=0, \quad v_{\mathrm{ac}, 2}=\frac{\partial u_{2}}{\partial t}=2 a \pi f \cos (2 \pi f t) \quad \text { on } \quad \Sigma_{\mathrm{ac}}
$$

in (1e), cf. [2].

\subsection{Finite Element Immersed Boundary method}

Red blood cells (RBCs) and malignant breast cancer cells (MCF-7) are viscoelastic bodies consisting of a cytoskeleton enclosing a heterogeneous cell interior [1]. In a simplified biomechanical model, neglecting viscoelastic properties, the cytoskeleton can be represented as an elastic membrane with specific elastic moduli and the cell interior as an incompressible fluid with specific density and viscosity. We further suppose that the density and viscosity are the same for the carrier fluid and the fluid enclosed by the membrane. This is a realistic assumption, since in the experiments the carrier fluid is manipulated by chemical additives to adjust these properties (density/viscosity matching). It also facilitates the application of the Immersed Boundary (IB) method. The IB method, which has been originally suggested by Peskin [20] (cf. also the survey article [21] and references therein), relies on the incompressible Navier-Stokes equations (1a)- $(1 \mathrm{~g})$ for the motion of the carrier fluid within an Eulerian coordinate system, equations describing the motion of the immersed cells within a Lagrangian coordinate system, and the interaction equations which transform Eulerian into Lagrangian quantities and vice versa. The finite element version of IB has been introduced in [5] and further studied in $[4,6,14]$.

We consider an immersed cell occupying a subdomain $B_{t}, t \in[0, T]$, with boundary $\partial B_{t}$ that is supposed to be a non-selfintersecting closed curve. We further assume that the boundary $\partial B_{0}$ of the initial configuration $B_{0}$ has length $L=\left|\partial B_{0}\right|$ and denote by $q \in[0, L]$ the Lagrangian coordinate labeling a material point on $\partial B_{0}$. We further refer to $\mathrm{X}(q, t)=\left(X_{1}(q, t), X_{2}(q, t)\right)^{T}$ as the position of that point at time $t \in(0, T]$ such that

$$
\mathrm{X} \in \mathrm{H}^{1}\left((0, T), \mathrm{L}^{2}([0, L])\right) \cap \mathrm{L}^{2}\left((0, T), \mathrm{H}_{\text {per }}^{3}([0, L])\right),
$$

where $\mathrm{H}_{\text {per }}^{3}([0, L])=\left\{\mathrm{Y} \in \mathrm{H}^{3}((0, L)): \partial^{k} \mathrm{Y}(0) / \partial q^{k}=\partial^{k} \mathrm{Y}(L) / \partial q^{k}, k=0,1,2\right\}$. The total elastic energy of the immersed boundary $\partial B_{t}$ is given by

$$
\begin{aligned}
E(t) & =E^{e}(t)+E^{b}(t), \quad t \in(0, T), \\
E^{e}(t) & =\int_{0}^{L} \mathcal{E}^{e}(\mathrm{X}(q, t)) d q, \quad E^{b}(t)=\int_{0}^{L} \varepsilon^{b}(\mathrm{X}(q, t)) d q,
\end{aligned}
$$


where $\mathcal{E}^{e}(t)$ and $\mathcal{E}^{b}(t)$ stand for the local energy densities,

$$
\mathcal{E}^{e}(\mathrm{X}(q, t))=\frac{\kappa_{e}}{2}\left(\left|\frac{\partial \mathrm{X}}{\partial q}(q, t)\right|^{2}-1\right), \quad \mathcal{E}^{b}(\mathrm{X}(q, t))=\frac{\kappa_{b}}{2}\left|\frac{\partial^{2} \mathrm{X}}{\partial q^{2}}(q, t)\right|^{2} .
$$

Here, $\kappa_{e}>0$ and $\kappa_{b}>0$ denote the elasticity coefficients with respect to elongation-compression (Young's modulus) and bending.

Denoting by $\mathrm{f}$ the local force density, $\mathrm{f}(q, t)=-E^{\prime}(\mathrm{X}(q, t))$, where $E^{\prime}$ stands for the Gâteaux derivative of $E$, the global force density $F$ in (1a) is given by

$$
\langle\mathrm{F}(t), \mathbf{w}\rangle_{\mathrm{H}^{-1}, \mathrm{H}_{0}^{1}}=\int_{0}^{L} \mathbf{f}(q, t) \cdot \mathbf{w}(\mathbf{X}(q, t)) d q, \quad \mathbf{w} \in \mathrm{H}_{0}^{1}(\Omega) .
$$

Remark 2.1.

For sufficiently smooth $\mathbf{w}$ in (5), integration by parts yields

$$
\begin{aligned}
\langle\mathrm{F}(t), \mathbf{w}\rangle_{\mathrm{H}^{-1}, \mathrm{H}_{0}^{1}}= & -\kappa_{e} \int_{0}^{L} \frac{\partial \mathrm{X}(\cdot, t)}{\partial q} \cdot \frac{\partial}{\partial q} \mathbf{w}(\mathrm{X}(\cdot, t)) d q-\kappa_{b} \int_{0}^{L} \frac{\partial^{2} \mathbf{X}(\cdot, t)}{\partial q^{2}} \cdot \frac{\partial^{2}}{\partial q^{2}} \mathbf{w}(\mathbf{X}(\cdot, t)) d q \\
= & -\kappa_{e} \int_{0}^{L} \frac{\partial \mathrm{X}(\cdot, t)}{\partial q} \cdot \mathbf{D}^{1} \mathbf{w}(\mathrm{X}(\cdot, t)) \frac{\partial \mathrm{X}(\cdot, t)}{\partial q} d q-\kappa_{b} \int_{0}^{L} \frac{\partial^{2} \mathrm{X}(\cdot, t)}{\partial q^{2}} \cdot \mathbf{D}^{1} \mathbf{w}(\mathrm{X}(\cdot, t)) \frac{\partial^{2} \mathrm{X}(\cdot, t)}{\partial q^{2}} d q \\
& -\kappa_{b} \int_{0}^{L} \frac{\partial^{2} \mathrm{X}(\cdot, t)}{\partial q^{2}} \cdot \mathbf{D}^{2} \mathbf{w}(\mathbf{X}(\cdot, t))\left(\frac{\partial \mathrm{X}(\cdot, t)}{\partial q}, \frac{\partial \mathrm{X}(\cdot, t)}{\partial q}\right) d q .
\end{aligned}
$$

Moreover, the immersed boundary moves with the velocity $v$ of the carrier fluid and hence, the equation of motion of the immersed boundary reads as follows:

$$
\begin{aligned}
\frac{\partial \mathrm{X}}{\partial t}(q, t) & =\mathrm{v}(\mathrm{X}(q, t), t), \quad(q, t) \in[0, L] \times(0, T], \\
\mathrm{X}(\cdot, 0) & =\mathrm{X}^{(0)}(\cdot) .
\end{aligned}
$$

\subsection{A stability estimate}

In case $\mathrm{v} \uparrow_{\Sigma}=0$, a stability estimate for the FE-IB has been derived in [6], whereas such an estimate has been provided in [14] for inflow and outflow boundary conditions of the form $\left.\boldsymbol{v}_{\Gamma_{\text {in }}} \cdot \mathbf{v}\right\rceil_{\Gamma_{\text {in }}}=-\boldsymbol{v}_{\Gamma_{\text {out }}} \cdot \mathbf{v} \Upsilon_{\Gamma_{\text {out }}}=g$ with $g$ being independent of $t \in[0, T]$. In the sequel, we will establish a stability estimate under the boundary conditions as given by (1c)-(1f).

We suppose that the inflow velocities $v_{\mathrm{in}}^{(i)}, 1 \leq i \leq 3$, in (1c) and $v_{\mathrm{ac}}$ in (1e) satisfy

$$
\begin{aligned}
& \mathrm{v}_{\mathrm{in}}^{(i)} \in \mathrm{H}^{1}\left((0, T), \mathrm{H}_{00}^{5 / 2+\mu}\left(\Gamma_{\mathrm{in}}^{(i)}\right)\right), \quad 1 \leq i \leq 3, \\
& \mathrm{v}_{\mathrm{ac}} \in \mathrm{H}^{1}\left((0, T), \mathrm{H}_{00}^{5 / 2+\mu}\left(\Gamma_{\mathrm{ac}}\right)\right),
\end{aligned}
$$

for some $\mu \in(0,1 / 2)$. We further construct $\mathrm{v}_{\text {out }}^{(i)}, 1 \leq i \leq 2$,

$$
\mathrm{v}_{\text {out }}^{(i)} \in \mathrm{H}^{1}\left((0, T), \mathrm{H}_{00}^{5 / 2+\mu}\left(\Gamma_{\text {out }}^{(i)}\right)\right), \quad 1 \leq i \leq 2,
$$

such that for $t \in[0, T]$ there holds

$$
\sum_{i=1}^{2} \int_{\Gamma_{\text {out }}^{(i)}} \mathbf{n} \cdot \mathbf{v}_{\text {out }}^{(i)}(s, t) d s+\sum_{i=1}^{3} \int_{\Gamma_{\text {in }}^{(i)}} \mathbf{n} \cdot \mathbf{v}_{\mathrm{in}}^{(i)}(s, t) d s+\int_{\Gamma_{\mathrm{ac}}} \mathbf{n} \cdot \mathbf{v}_{\mathrm{ac}}(s, t) d s=0 .
$$




\section{Lemma 2.2.}

Under the assumptions (8a), (8b), (9), and (10) there exists a function $\widehat{\psi} \in \mathrm{H}^{1}\left((0, T), \mathbf{H}^{3+\mu}(\Omega) \cap \mathrm{H}\left(\operatorname{div}^{0}, \Omega\right)\right)$ satisfying for $t \in[0, T]$,

$$
\begin{array}{ll}
\widehat{\psi}(\cdot, t) \Gamma_{\Gamma_{\text {in }}^{(i)}}=\mathrm{v}_{\Gamma_{\text {in }}^{(i)}}(\cdot, t), & 1 \leq i \leq 3, \\
\widehat{\psi}(\cdot, t) \Gamma_{\Gamma_{\text {out }}^{(i)}}=\mathrm{v}_{\Gamma_{\text {out }}^{(i)}}(\cdot, t), & 1 \leq i \leq 2, \\
\widehat{\psi}(\cdot, t) \Gamma_{\Gamma_{\text {ac }}}=v_{\Gamma_{\text {ac }}}(\cdot, t) . &
\end{array}
$$

Moreover, there exist constants $\widehat{C}_{1}>0$ and $\widehat{C}_{2}>0$ such that for $t \in[0, T]$ it holds

$$
\begin{aligned}
\|\widehat{\psi}(\cdot, t)\|_{C^{2, \mu}(\bar{\Omega})} & \leq \widehat{C}_{1} g_{1}(t), \\
\int_{0}^{t}\left\|\frac{\partial \widehat{\psi}}{\partial \tau}(\cdot, \tau)\right\|_{C^{2, \mu}(\bar{\Omega})}^{2} d \tau & \leq \widehat{C}_{2} g_{2}(t),
\end{aligned}
$$

where the upper bounds $g_{1}(t)$ and $g_{2}(t)$ are given by

$$
\begin{aligned}
& g_{1}(t)=\sum_{i=1}^{3}\left\|v_{\text {in }}^{(i)}(\cdot, t)\right\|_{\mathrm{H}_{00}^{5 / 2+\mu}\left(\Gamma_{\text {in }}^{(i)}\right)}+\sum_{i=1}^{2}\left\|v_{\text {out }}^{(i)}(\cdot, t)\right\|_{\mathrm{H}_{00}^{5 / 2+\mu}\left(\Gamma_{\text {out }}^{(i)}\right)}+\left\|v_{\text {ac }}(\cdot, t)\right\|_{\mathrm{H}_{00}^{5 / 2+\mu}\left(\Gamma_{\text {ac }}\right)^{\prime}} \\
& g_{2}(t)=\int_{0}^{t}\left(\sum_{i=1}^{3}\left\|\frac{\partial v_{\text {in }}^{(i)}}{\partial \tau}(\cdot, \tau)\right\|_{\mathrm{H}_{00}^{5 / 2+\mu}\left(\Gamma_{\text {in }}^{(i)}\right)}^{2}+\sum_{i=1}^{2}\left\|\frac{\partial v_{\text {out }}^{(i)}}{\partial \tau}(\cdot, \tau)\right\|_{\mathrm{H}_{00}^{5 / 2+\mu}\left(\Gamma_{\text {out }}^{(i)}\right)}^{2}+\left\|\frac{\partial v_{\mathrm{ac}}}{\partial \tau}(\cdot, \tau)\right\|_{\mathrm{H}_{00}^{5 / 2+\mu}\left(\Gamma_{\mathrm{ac}}\right)}^{2}\right) d \tau \text {. }
\end{aligned}
$$

Proof. We denote by $\widetilde{v}_{\mathrm{in}}^{(i)}(\cdot, t) \in \mathbf{H}^{5 / 2+\mu}(\Gamma), 1 \leq i \leq 3, \widetilde{\mathrm{v}}_{\mathrm{out}}^{(i)}(\cdot, t) \in \mathbf{H}^{5 / 2+\mu}(\Gamma), 1 \leq i \leq 2$, and $\widetilde{\mathrm{v}}_{\mathrm{ac}}(\cdot, t) \in \mathbf{H}^{5 / 2+\mu}(\Gamma)$ the extensions of $v_{\text {in }}^{(i)}(\cdot, t), v_{\text {out }}^{(i)}(\cdot, t)$, and $v_{\text {ac }}(\cdot, t)$ by zero to $\Gamma$ such that

$$
\begin{aligned}
\left\|\widetilde{v}_{\text {in }}^{(i)}(\cdot, t)\right\|_{5 / 2+\mu, \Gamma} & \lesssim\left\|v_{\text {in }}^{(i)}(\cdot, t)\right\|_{\mathrm{H}_{00}^{5 / 2+\mu}\left(\Gamma_{\text {in }}^{(i)}\right)}, \\
\left\|\widetilde{\mathrm{v}}_{\text {out }}^{(i)}(\cdot, t)\right\|_{5 / 2+\mu, \Gamma} & \lesssim\left\|v_{\text {out }}^{(i)}(\cdot, t)\right\|_{\mathrm{H}_{00}^{5 / 2+\mu}\left(\Gamma_{\text {out }}^{(i)}\right)^{\prime}}, \\
\left\|\widetilde{v}_{\text {ac }}(\cdot, t)\right\|_{5 / 2+\mu, \Gamma} & \lesssim\left\|\mathrm{v}_{\text {ac }}(\cdot, t)\right\|_{\mathrm{H}_{00}^{5 / 2+\mu}(\Gamma \text { ac })} .
\end{aligned}
$$

We define $\widetilde{\mathbf{v}} \in \mathbf{H}^{1}\left((0, T), \mathbf{H}^{5 / 2+\mu}(\Gamma)\right)$ by

$$
\widetilde{\mathbf{v}}=\sum_{i=1}^{3} \widetilde{\mathbf{v}}_{\text {in }}^{(i)}+\sum_{i=1}^{2} \widetilde{\mathbf{v}}_{\text {out }}^{(i)}+\widetilde{\mathbf{v}}_{\mathrm{ac}}
$$

and observe that due to (10) there holds

$$
\int_{\Gamma}(\mathbf{n} \cdot \widetilde{\mathbf{v}}) d s=0
$$

In view of the trace theorem [30] and (14) there exists $\widehat{\psi} \in \mathbf{H}^{1}\left((0, T), \mathbf{H}^{3+\mu}(\Omega) \cap \mathbf{H}\left(\operatorname{div}^{0}, \Omega\right)\right)$ satisfying (11a)-(11c) and

$$
\|\widehat{\psi}(\cdot, t)\|_{3+\mu, \Omega} \lesssim \sum_{i=1}^{3}\left\|\widetilde{\mathrm{v}}_{\mathrm{in}}^{(i)}\right\|_{5 / 2+\mu, \Gamma}+\sum_{i=1}^{2}\left\|\widetilde{\mathbf{v}}_{\mathrm{out}}^{(i)}\right\|_{5 / 2+\mu, \Gamma}+\left\|\widetilde{\mathbf{v}}_{\mathrm{ac}}\right\|_{5 / 2+\mu, \Gamma} .
$$

Finally, (12a) and (12b) follow from the continuous embedding of $H^{3+\mu}(\Omega)$ in $C^{2, \mu}(\bar{\Omega})$. 
For the proof of the subsequent energy estimate, we note that the computational domain $\Omega \subset \mathbb{R}^{2}$ is such that for $\mathrm{v} \in \mathrm{W}(0, T)$ the Poincaré-Friedrichs inequality

$$
\|\mathbf{v}(\cdot, t)\|_{0, \Omega} \leq C_{\Omega}\left(\|\nabla \mathbf{v}(\cdot, t)\|_{0, \Omega}^{2}+\|\mathbf{v}(\cdot, t)\|_{0, \Gamma}^{2}\right)^{1 / 2}, \quad t \in[0, T]
$$

holds true for some constant $C_{\Omega}>0$.

Theorem 2.3.

Suppose that the data of the problem satisfy (8a) and (8b), and that the additional assumption

$$
\max _{0 \leq t \leq T} g_{1}(t) \leq \frac{\eta}{8 \rho \widehat{C}_{1} C_{\Omega}^{2}}
$$

holds true, where $g_{1}(t), t \in[0, T]$, is from (13a) and the positive constants $\widehat{C}_{1}, C_{\Omega}$ are given by (12a) and (15). Moreover, assume that the triple $(\mathrm{v}, p, \mathrm{X})$ satisfies (2a)-(2c) and (7a) and (7b). Then, there exists a positive constant $C$, depending on $\rho, \eta, \kappa_{e}, \kappa_{b}, \widehat{C}_{i}, 0 \leq i \leq 2$, and $C_{\Omega}$ such that

$$
\begin{aligned}
\frac{\rho}{4}\|\mathbf{v}(\cdot, t)\|_{0, \Omega}^{2} & +\frac{\eta}{8} \int_{0}^{t}\|\nabla \mathbf{v}(\cdot, t)\|_{0, \Omega}^{2} d \tau+\frac{\kappa_{e}}{2}\left\|\frac{\partial \mathbf{X}(\cdot, t)}{\partial q}\right\|_{0,[0, L]}^{2}+\frac{\kappa_{b}}{2}\left\|\frac{\partial^{2} \mathbf{X}(\cdot, t)}{\partial q^{2}}\right\|_{0,[0, L]}^{2} \\
\leq & C\left(g_{1}(0)^{2}+g_{1}(t)^{2}+\int_{0}^{t}\left(g_{1}(\tau)^{2}+g_{2}(\tau)^{2}\right) d \tau+\max _{0 \leq \tau \leq t} g_{1}(\tau) \int_{0}^{t}\|\mathbf{v}(\cdot, \tau)\|_{0, \Gamma}^{2} d \tau+\left\|\mathbf{u}^{(0)}\right\|_{0, \Omega}^{2}\right. \\
& \left.+\left\|\frac{\partial \mathbf{X}^{(0)}}{\partial q}\right\|_{0,[0, L]}^{2}+\left\|\frac{\partial^{2} \mathbf{X}^{(0)}}{\partial q^{2}}\right\|_{0,[0, L]}^{2}+\int_{0}^{t}\left\|\frac{\partial \mathbf{X}(\cdot, \tau)}{\partial q}\right\|_{0,[0, L]}^{2} d \tau+\int_{0}^{t}\left\|\frac{\partial^{2} \mathbf{X}(\cdot, \tau)}{\partial q^{2}}\right\|_{0,[0, L]}^{2} d \tau\right) .
\end{aligned}
$$

Proof. Due to Lemma 2.2, $\mathbf{w}=\mathbf{v}-\widehat{\psi}$ is an admissible test function in (2a). Integrating over $[0, t]$, it follows that

$$
\int_{0}^{t}\left\langle\rho \frac{\partial \mathrm{v}}{\partial \tau}, \mathbf{v}-\widehat{\psi}\right\rangle d \tau+\int_{0}^{t} a(\mathrm{v}, \mathrm{v}-\widehat{\psi}) d \tau=\int_{0}^{t}\langle\mathrm{~F}(\tau), \mathrm{v}-\widehat{\psi}\rangle_{\mathrm{H}^{-1}, \mathrm{H}_{0}^{1}} d \tau
$$

Using partial integration, the Cauchy-Schwarz inequality, Young's inequality with $\varepsilon_{1}>0$, and the Poincaré-Friedrichs inequality (15), the first term on the left-hand side in (18) can be bounded from below according to

$$
\begin{aligned}
\int_{0}^{t}\left\langle\rho \frac{\partial \mathrm{v}}{\partial \tau}, \mathrm{v}-\widehat{\psi}\right\rangle_{\mathrm{H}^{-1}, \mathrm{H}_{0}^{1}} d \tau= & \frac{\rho}{2} \int_{0}^{t} \frac{\partial}{\partial \tau}\|\mathrm{v}(\tau)\|_{0, \Omega}^{2} d \tau-\rho(\mathrm{v}(\cdot, t), \widehat{\psi}(\cdot, t))_{0, \Omega}+\rho(\mathrm{v}(\cdot, 0), \widehat{\psi}(\cdot, 0))_{0, \Omega} \\
& +\rho \int_{0}^{t}\left(\mathrm{v}(\cdot, \tau), \frac{\partial \widehat{\psi}}{\partial \tau}(\cdot, \tau)\right)_{0, \Omega} d \tau \\
\geq \frac{\rho}{4}\|\mathbf{v}(\cdot, t)\|_{0, \Omega}^{2}-\frac{3 \rho}{4}\|\mathbf{v}(\cdot, 0)\|_{0, \Omega}^{2}-\rho\left(\|\widehat{\psi}(\cdot, t)\|_{0, \Omega}^{2}+\|\widehat{\psi}(\cdot, 0)\|_{0, \Omega}^{2}\right) & \\
& -\varepsilon_{1} \rho \int_{0}^{t}\|\mathbf{v}(\cdot, \tau)\|_{0, \Omega}^{2} d \tau-\frac{\rho}{4 \varepsilon_{1}} \int_{0}^{t}\left\|\frac{\partial \widehat{\psi}}{\partial \tau}(\cdot, \tau)\right\|_{0, \Omega}^{2} d \tau \\
\geq & \frac{\rho}{4}\|\mathbf{v}(\cdot, t)\|_{0, \Omega}^{2}-\frac{3 \rho}{4}\|\mathbf{v}(\cdot, 0)\|_{0, \Omega}^{2}-\rho\left(\|\widehat{\psi}(\cdot, t)\|_{0, \Omega}^{2}+\|\widehat{\psi}(\cdot, 0)\|_{0, \Omega}^{2}\right) \\
& -\varepsilon_{1} \rho C_{\Omega}^{2}\left(\int_{0}^{t}\|\nabla \mathbf{v}(\cdot, \tau)\|_{0, \Omega}^{2} d \tau+\int_{0}^{t}\|\mathbf{v}(\cdot, \tau)\|_{0, \Gamma}^{2} d \tau\right)-\frac{\rho}{4 \varepsilon_{1}} \int_{0}^{t}\left\|\frac{\partial \widehat{\psi}}{\partial \tau}(\cdot, \tau)\right\|_{0, \Omega}^{2} d \tau .
\end{aligned}
$$


For the second term on the left-hand side in (18) we obtain

$$
\int_{0}^{t} a(\mathbf{v}, \mathbf{v}-\widehat{\psi}) d \tau=\int_{0}^{t}(\rho(\mathbf{v} \cdot \nabla) \mathbf{v}, \mathbf{v})_{0, \Omega} d \tau-\int_{0}^{t}(\rho(\mathbf{v} \cdot \nabla) \mathbf{v}, \widehat{\psi})_{0, \Omega} d \tau+\int_{0}^{t}(\eta \nabla \mathbf{v}, \nabla(\mathbf{v}-\widehat{\psi}))_{0, \Omega} d \tau .
$$

The first term on the right-hand side of (20) can be estimated as follows:

$$
\begin{aligned}
\int_{0}^{t}(\rho(\mathbf{v} \cdot \nabla) \mathrm{v}, \mathrm{v})_{0, \Omega} d \tau & =\frac{\rho}{2} \int_{0}^{t} \int_{\Gamma}|\mathbf{v}(s, \tau)|^{2} \mathbf{n} \cdot \mathbf{v}(s, \tau) d s d \tau \\
& \geq-\frac{\rho}{2} \int_{0}^{t} \int_{\Gamma}|\mathbf{v}(s, \tau)|^{2}|\mathbf{n} \cdot \mathbf{v}(s, \tau)| d s d \tau \geq-\frac{\rho}{2} \max _{0 \leq \tau \leq t} g_{1}(t) \int_{0}^{t}\|\mathbf{v}(\cdot, \tau)\|_{0, \Gamma}^{2} d \tau .
\end{aligned}
$$

By means of the Cauchy-Schwarz inequality, Young's inequality, and the Poincaré-Friedrichs inequality (15), the remaining two terms on the right-hand side in (20) can be estimated from below according to

$$
\begin{aligned}
& \int_{0}^{t}(\eta \nabla \mathbf{v}, \nabla(\mathbf{v}-\widehat{\psi}))_{0, \Omega} d \tau-\int_{0}^{t}(\rho(\mathbf{v} \cdot \nabla) \mathbf{v}, \widehat{\psi})_{0, \Omega} d \tau \\
& \geq \eta \int_{0}^{t}\|\nabla \mathbf{v}(\cdot, \tau)\|_{0, \Omega}^{2} d \tau-\eta \int_{0}^{t}\|\nabla \mathbf{v}(\cdot, \tau)\|_{0, \Omega}\|\nabla \widehat{\psi}(\cdot, \tau)\|_{0, \Omega} d \tau \\
& \quad-\rho \widehat{C}_{1} \max _{0 \leq \tau \leq t} g_{1}(\tau) \int_{0}^{t} \sum_{i, j=1}^{2}\left\|\mathbf{v}_{j}(\cdot, \tau)\right\|_{0, \Omega}\left\|\frac{\partial \mathbf{v}_{i}}{\partial x_{j}}(\cdot, \tau)\right\|_{0, \Omega} d \tau \\
& \geq\left(\frac{\eta}{2}-\rho \sqrt{2} \widehat{C}_{1} C_{\Omega}^{2} \max _{0 \leq \tau \leq t} g_{1}(\tau)\right) \int_{0}^{t}\|\nabla \mathbf{v}(\cdot, \tau)\|_{0, \Omega}^{2} d \tau-\frac{\rho \sqrt{2}}{2} C_{\Omega}^{2} \max _{0 \leq \tau \leq t} g_{1}(\tau) \int_{0}^{t}\|\mathbf{v}(\cdot, \tau)\|_{0, \Gamma}^{2} d \tau-\frac{\eta}{2} \int_{0}^{t} g_{1}^{2}(\tau) d \tau .
\end{aligned}
$$

In view of (6) and (7a), for the right-hand side in (18) we find

$$
\begin{aligned}
\int_{0}^{t}\langle\mathrm{~F}(\tau), \mathbf{v}\rangle_{\mathrm{H}^{-1}, \mathrm{H}_{0}^{1}} d \tau & =\int_{0}^{t}\left(-\kappa_{e} \int_{0}^{L} \frac{\partial \mathrm{X}(q, \tau)}{\partial q} \cdot \frac{\partial}{\partial q} \mathbf{v}(\mathrm{X}(q, \tau)) d q-\kappa_{b} \int_{0}^{L} \frac{\partial^{2} \mathrm{X}(q, \tau)}{\partial q^{2}} \cdot \frac{\partial^{2}}{\partial q^{2}} \mathbf{v}(\mathrm{X}(q, \tau)) d q\right) d \tau \\
& =\int_{0}^{t}\left(-\kappa_{e} \int_{0}^{L} \frac{\partial \mathrm{X}(q, \tau)}{\partial q} \cdot \frac{\partial}{\partial \tau}\left(\frac{\partial \mathrm{X}(q, \tau)}{\partial q}\right) d q-\kappa_{b} \int_{0}^{L} \frac{\partial^{2} \mathrm{X}(q, \tau)}{\partial q^{2}} \cdot \frac{\partial}{\partial \tau}\left(\frac{\partial^{2} \mathrm{X}(q, \tau)}{\partial q^{2}}\right) d q\right) d \tau \\
& =-\frac{\kappa_{e}}{2} \int_{0}^{t} \frac{\partial}{\partial \tau}\left\|\frac{\partial \mathrm{X}}{\partial q}(\tau)\right\|_{0,[0, L]}^{2} d \tau-\frac{\kappa_{b}}{2} \int_{0}^{t} \frac{\partial}{\partial \tau}\left\|\frac{\partial^{2} \mathrm{X}}{\partial q^{2}}(\tau)\right\|_{0,[0, L]}^{2} d \tau \\
& =\frac{\kappa_{e}}{2}\left(\left\|\frac{\partial \mathrm{X}}{\partial q}(0)\right\|_{0,[0, L]}^{2}-\left\|\frac{\partial \mathrm{X}}{\partial q}(t)\right\|_{0,[0, L]}^{2}\right)+\frac{\kappa_{b}}{2}\left(\left\|\frac{\partial^{2} \mathrm{X}}{\partial q^{2}}(0)\right\|_{0,[0, L]}^{2}-\left\|\frac{\partial^{2} \mathrm{X}}{\partial q^{2}}(t)\right\|_{0,[0, L]}^{2}\right) .
\end{aligned}
$$

Using (6) again, we get

$$
\begin{aligned}
& \int_{0}^{t}\langle\mathrm{~F}(\tau), \widehat{\psi}\rangle_{\mathrm{H}^{-1}, \mathrm{H}_{0}^{1}} d \tau=\kappa_{e} \int_{0}^{t}\left(\frac{\partial \mathrm{X}}{\partial q}, \mathrm{D}^{1} \widehat{\psi}(\mathrm{X}(\cdot, \tau)) \frac{\partial \mathrm{X}}{\partial q}\right)_{0,[0, L]} d \tau+\kappa_{b} \int_{0}^{t}\left(\frac{\partial^{2} \mathrm{X}}{\partial q^{2}}, \mathrm{D}^{1} \widehat{\psi}(\mathrm{X}(\cdot, \tau)) \frac{\partial^{2} \mathrm{X}}{\partial q^{2}}\right)_{0,[0, L]} d \tau \\
& +\kappa_{b} \int_{0}^{t}\left(\frac{\partial^{2} \mathrm{X}}{\partial q^{2}}, \mathrm{D}^{2} \widehat{\psi}(\mathrm{X}(\cdot, \tau))\left(\frac{\partial \mathrm{X}}{\partial q}, \frac{\partial \mathrm{X}}{\partial q}\right)\right)_{0,[0, L]} d \tau
\end{aligned}
$$

The stability estimate (17) now follows by using (19) with $\varepsilon_{1}=\eta /\left(4 \rho C_{\Omega}^{2}\right)$ and (20)-(24) in (18). 


\section{Remark 2.4.}

The meaning of the assumption (16) is that the magnitude of the inflow velocities should be bounded depending on the fluid parameters $\rho$ and $\eta$ as well as the geometry of the channel as reflected by the parameters $\widehat{C}$ and $C_{\Omega}$. Actually, the inflow velocities used in our simulations, cf. Section 4, and the data are such that (16) is always satisfied. A violation of (16) corresponds to the experimentally observable scenario that extremely high inflow velocities cause the immersed membranes to get torn apart.

\section{Semi-Implicit FE Immersed Boundary method}

For discretization in space and time we use the Backward Euler/Forward Euler Finite Element Immersed Boundary method from [14] in the sense that we discretize the Navier-Stokes equations by the backward Euler method in time and by Taylor-Hood P2/P1 elements in space, whereas we discretize the equation of motion of the immersed cell by the forward Euler scheme in time and by periodic cubic splines in space.

\subsection{Discretization in space and time}

Let $\mathcal{T}_{h}(\Omega)$ be a quasi-uniform simplicial triangulation of $\Omega$ that aligns with the partition of $\Gamma$. For $K \in \mathcal{T}_{h}(\Omega)$, we denote by $|K|$ the area of $K$, by $h_{K}$ the diameter of $K$, and we set $h=\max \left\{h_{K}: K \in \mathcal{T}_{h}(\Omega)\right\}$. Further, $P_{k}(K), k \in \mathbb{N}$, refers to the set of polynomials of degree $\leq k$ on $K$. For the spatial discretization of the weak formulation (2a) $-(2 c)$ of the incompressible Navier-Stokes equations we use P2/P1 Taylor-Hood elements [7], i.e., we define

$$
\mathrm{V}_{h}=\left\{\mathrm{v}_{h} \in \mathrm{C}(\bar{\Omega}): \mathrm{v}_{h} \uparrow_{K} \in P_{2}(K)^{2}, K \in \mathcal{T}_{h}(\Omega)\right\}, \quad Q_{h}=\left\{w_{h} \in C(\bar{\Omega}):\left.w_{h}\right|_{K} \in P_{1}(K), K \in \mathcal{T}_{h}(\Omega)\right\}
$$

and set $\mathrm{V}_{h, \Gamma_{\text {in }}^{(i)}}=\mathrm{V}_{h} \Gamma_{\Gamma_{\text {in }}^{(i)}}, 1 \leq i \leq 2, \mathrm{~V}_{h, \Gamma_{\mathrm{ac}}}=\mathrm{V}_{h} \Gamma_{\Gamma_{\mathrm{ac}}}$. The finite element spaces are spanned by the canonically specified nodal basis functions.

For the discretization in time we consider an equidistant partition

$$
\mathcal{T}_{\Delta t}=\left\{0=t_{0}<t_{1}<\ldots<t_{M}=T\right\}, \quad M \in \mathbb{N},
$$

of the time interval $[0, T]$ into subintervals of length $\Delta t=T / M$ and denote by $\mathrm{v}_{h}^{(m)}$ an approximation of $\mathrm{v}_{h} \in \mathrm{V}_{h}$ at $t=t_{m}$. We further refer to $\mathrm{D}_{\Delta t}^{+} \mathbf{v}_{h}^{(m)}=\left(\mathbf{v}_{h}^{(m+1)}-\mathbf{v}_{h}^{(m)}\right) / \Delta t$ and $\mathrm{D}_{\Delta t}^{-} \mathbf{v}_{h}^{(m)}=\left(\mathbf{v}_{h}^{(m)}-\mathbf{v}_{h}^{(m-1)}\right) / \Delta t$ as the forward and backward difference operator. For $t_{m} \in \mathcal{T}_{\Delta t}$, we define $\mathbf{v}_{h, \text { in }}^{(i)}\left(\cdot, t_{m}\right), 1 \leq i \leq 2$, and $\mathbf{v}_{h, \text { ac }}\left(\cdot, t_{m}\right)$ as the $L^{2}$-projection of $\mathbf{v}_{\text {in }}^{(i)}\left(\cdot, t_{m}\right)$ onto $\mathrm{V}_{h} \Gamma_{\Gamma_{i \mathrm{in}}^{(i)}}$ and of $\mathrm{v}_{\mathrm{ac}}\left(\cdot, t_{m}\right)$ onto $\mathrm{V}_{h} \uparrow_{\Gamma_{\mathrm{ac}}}$. We set

$$
\begin{aligned}
\mathbf{W}_{h}^{(m)} & =\left\{\mathbf{w}_{h}^{(m)} \in \mathbf{C}(\bar{\Omega}): \mathbf{w}_{h}^{(m)} \in \mathbf{V}_{h}, \mathbf{w}_{h}^{(m)} \Gamma_{\Gamma_{\text {in }}^{(i)}}=\mathbf{v}_{h \text { in }}^{(i)}\left(\cdot, t_{m}\right), 1 \leq i \leq 2, \mathbf{w}_{h}^{(m)} \Gamma_{\Gamma_{\mathrm{ac}}}=\mathbf{v}_{h, \text { ac }}\left(\cdot, t_{m}\right), \mathbf{w}_{h}^{(m)} \Gamma_{\Gamma_{D}}=0\right\}, \\
Q_{h}^{(m)} & =\left\{w_{h}^{(m)} \in C(\bar{\Omega}): w_{h}^{(m)} \Gamma_{K} \in Q_{h}\right\} .
\end{aligned}
$$

The discretization of the immersed boundary is done with respect to a partition

$$
\mathcal{T}_{\Delta q}=\left\{0=q_{0}<q_{1}<\ldots<q_{R}=L\right\}, \quad R \in \mathbb{N},
$$

of the interval $[0, L]$ into subintervals $l_{i}=\left[q_{r-1}, q_{r}\right], 1 \leq r \leq R$, of length $\Delta q_{r}=q_{r}-q_{r-1}$ with $\Delta q=$ $\max \left\{\Delta q_{r}: 1 \leq r \leq R\right\}$. We approximate $X$ from (4) by periodic cubic splines

$$
\mathrm{S}_{h}=\left\{\mathrm{Y}_{h} \in \mathrm{C}^{2}([0, L] ; \Omega): \mathrm{Y}_{h} \uparrow_{l_{r}} \in P_{3}\left(I_{r}\right)^{2}, 1 \leq r \leq R, \mathrm{Y}_{h}^{(k)}\left(q_{0}\right)=\mathrm{Y}_{h}^{(k)}\left(q_{R}\right), k=0,1,2\right\}
$$


where $P_{3}\left(I_{r}\right)$ stands for the set of polynomials of degree $\leq 3$ on $I_{r}$. For $\mathrm{Y}_{h} \in \mathrm{S}_{h}$, we set $\mathrm{Y}_{h, r}=\mathrm{Y}_{h}\left(q_{r}\right), 0 \leq r \leq R$. The discrete immersed cell occupies subdomains $B_{h, t_{m}} \subset \Omega$ with boundaries $\partial B_{h, t_{m}}$ that are $C^{2}$ curves described by the periodic cubic spline $\mathbf{X}_{h}^{(m)}(\cdot) \in \mathbf{S}_{h}$.

We define the total discrete energy by means of $E_{h}\left(t_{m}\right)=E_{h}^{e}\left(t_{m}\right)+E_{h}^{b}\left(t_{m}\right)$, where the discrete elastic energy $E_{h}^{e}\left(t_{m}\right)$ and the discrete bending energy $E_{h}^{b}\left(t_{m}\right)$ are given by

$$
E_{h}^{e}\left(t_{m}\right)=\frac{\kappa_{e}}{2} \int_{0}^{L}\left(\left|\frac{\partial \mathrm{X}_{h}^{(m)}}{\partial q}(q)\right|^{2}-1\right) d q, \quad E_{h}^{b}\left(t_{m}\right)=\frac{\kappa_{b}}{2} \sum_{r=1}^{R} \int_{q_{r-1}}^{q_{r}}\left|\frac{\partial^{2} \mathbf{X}_{h}^{(m)}}{\partial q^{2}}(q)\right|^{2} d q
$$

Observing that $\partial^{3} \mathbf{X}_{h}^{(m)}(q) / \partial q^{3}$ is constant on $I_{r}$, the discrete force density takes the form

$$
\begin{aligned}
\left\langle\mathbf{F}_{h}^{(m)}, \mathbf{w}_{h}\right\rangle_{h} & =-\kappa_{e} \int_{0}^{L} \frac{\partial \mathbf{X}_{h}^{(m)}}{\partial q} \cdot \frac{\partial}{\partial q} \mathbf{w}_{h}\left(\mathbf{X}_{h}^{(m)}(q)\right) d q+\kappa_{b} \sum_{i=1}^{R} \int_{q_{i-1}}^{q_{i}} \frac{\partial^{3} \mathbf{X}_{h}^{(m)}}{\partial q^{3}} \cdot \frac{\partial}{\partial q} \mathbf{w}_{h}\left(\mathbf{X}_{h}^{(m)}(q)\right) d q \\
& \left.=-\kappa_{e} \int_{0}^{L} \frac{\partial \mathbf{X}_{h}^{(m)}(q)}{\partial q} \cdot \nabla \mathbf{w}_{h}\left(\mathbf{X}_{h}^{(m)}(q)\right) \frac{\partial \mathbf{X}_{h}^{(m)}}{\partial q} d q+\kappa_{b} \sum_{r=1}^{R} \frac{\partial^{3} \mathbf{X}_{h}^{(m)}}{\partial q^{3}}\right\rceil_{l_{r}} \int_{q_{r-1}}^{q_{r}} \nabla \mathbf{w}_{h}\left(\mathbf{X}_{h}^{(m)}(q)\right) \frac{\partial \mathbf{X}_{h}^{(m)}}{\partial q} d q,
\end{aligned}
$$

which is a discrete approximation of (6).

The Backward Euler/Forward Euler FE-IB reads as follows: Given $\mathbf{v}_{h}^{(0)} \in \mathbf{W}_{h}^{(0)}$ and $\mathrm{X}_{h}^{(0)} \in \mathrm{S}_{h}$, for $m=0, \ldots, M-1$ we perform the following two steps:

Step 1: Compute $\left(\mathbf{v}_{h}^{(m+1)}, p_{h}^{(n+1)}\right) \in \mathbf{W}_{h}^{(m+1)} \times Q_{h}^{(m+1)}$ such that for all $\mathbf{w}_{h} \in \mathbf{V}_{h, 0}$,

$$
\begin{aligned}
\left(\rho \mathbf{D}_{\Delta t}^{+} \mathbf{v}_{h}^{(m)}, \mathbf{w}_{h}\right)_{0, \Omega}+a\left(\mathbf{v}_{h}^{(m+1)}, \mathbf{w}_{h}\right)-b\left(p_{h}^{(m+1)}, \mathbf{w}_{h}\right) & =\ell_{h}^{(m)}\left(\mathbf{w}_{h}\right) \\
b\left(w_{h}, \mathbf{v}_{h}^{(m+1)}\right) & =0
\end{aligned}
$$

where $\ell_{h}^{(m)}\left(\mathbf{w}_{h}\right)=\left\langle\mathbf{F}_{h}^{(m)}, \mathbf{w}_{h}\right\rangle_{h}$ is given by (25).

Step 2: Compute $\mathbf{X}_{h}^{(m+1)} \in \mathrm{S}_{h}$ according to

$$
\mathrm{D}_{\Delta t}^{+} \mathbf{X}_{r}^{(m)}=\mathbf{v}_{h}^{(m+1)}\left(\mathbf{X}_{r}^{(m)}\right), \quad 1 \leq r \leq R
$$

\subsection{Stability of the semi-implicit FE-IB}

For the derivation of a stability estimate for the semi-implicit Backward Euler/Forward Euler FE-IB we note that the boundary $\partial B_{h, t_{m}}$ of the immersed cell at time $t_{m}$ consists of $C^{2}$ segments $\partial B_{h, t_{m}}^{(r)}$ connecting the material points $X_{h, r-1}^{(m)}$ and $\mathbf{X}_{h, r}^{(m)}, 1 \leq r \leq R$. Referring to $\mathcal{T}_{h}\left(\partial B_{h, t_{m}}^{(r)}\right)$ as the set $\left\{K \in \mathcal{T}_{h}(\Omega): K \cap \partial B_{h, t_{m}}^{(r)} \neq \emptyset\right\}$ we have

$$
\left\|\nabla \mathbf{v}_{h}^{(m+1)}\right\|_{0, \partial B_{h, t_{m}}^{(r)}}^{2} \leq \sum_{K \in \mathcal{T}_{h}\left(\partial B_{h, t_{m}}^{(r)}\right)} C_{K} h_{K}^{-1}\left\|\nabla \mathbf{v}_{h}^{(m+1)}\right\|_{0, K^{\prime}}^{2}
$$

where $C_{K}$ is a positive constant independent of $h_{K}$. Due to the quasi-uniformity of $\mathcal{T}_{h}(\Omega)$ there exist constants $0<c_{Q} \leq$ $C_{Q}$, that only depend on the local geometry of the triangulation, such that

$$
c_{Q} h \leq h_{K} \leq C_{Q} h, \quad K \in \mathcal{T}_{h}(\Omega)
$$


Hence, denoting by $C_{r}^{(m)}$ the maximum number of $C^{2}$ curve segments contained in an element $K \in \mathcal{T}_{h}\left(\partial B_{h, t_{m}}^{(r)}\right)$ and setting

$$
C_{\text {cell }}=C_{Q}^{-1} \max _{0 \leq m \leq M-1} \max _{1 \leq r \leq R}\left(C_{r}^{(m)} \max _{K \in \mathcal{T}_{h}\left(\partial B_{h, t_{m}}^{(r)}\right)} C_{K}\right)
$$

from (28) we obtain

$$
\left\|\nabla \mathbf{v}_{h}^{(m+1)}\right\|_{0, \partial B_{h, t_{m}}}^{2} \leq C_{\text {cell }} h^{-1}\left\|\nabla \mathbf{v}_{h}^{(m+1)}\right\|_{0, \Omega}^{2}
$$

In view of $\partial \mathrm{X}_{h}^{(m)} / \partial q \in \mathrm{C}^{1}([0, L]), 0 \leq m \leq M$, and taking into account that the third derivatives $\partial^{3} \mathrm{X}_{h}^{(m)} / \partial q^{3}$ are constant vectors on $I_{r}, 1 \leq r \leq R$, we further define

$$
\Lambda_{1}=\max _{0 \leq m \leq M} \max _{q \in[0, L)}\left|\frac{\partial \mathbf{X}_{h}^{(m)}}{\partial q}\right|, \quad \Lambda_{2}=\max _{0 \leq m \leq M} \max _{1 \leq r \leq R}\left|\frac{\partial^{3} \mathbf{X}_{h}^{(m)}}{\partial q^{3}} \Upsilon_{I_{r}}\right|
$$

Moreover, we refer to $\widehat{\psi}_{h}\left(\cdot, t_{m}\right)$ as the biquadratic spline interpolant of $\widehat{\psi}\left(\cdot, t_{m}\right)$ from Lemma 2.2. Then, there exist constants $C_{2}^{(k)}>0$ such that

$$
\begin{aligned}
\left\|\mathrm{D}^{k} \widehat{\psi}_{h}\left(\cdot, t_{m}\right)\right\|_{C^{k}(\bar{\Omega})} \leq C_{2}^{(k)} g_{1}^{(m)}, & 0 \leq k \leq 2, \\
\sum_{\ell=0}^{m-1}\left\|\mathrm{D}_{\Delta t}^{+} \widehat{\psi}_{h}\left(\cdot, t_{\ell}\right)\right\|_{C^{k}(\bar{\Omega})} \leq C_{2}^{(k)} g_{2}^{(m)}, & 0 \leq k \leq 2 .
\end{aligned}
$$

Here, $g_{1}^{(m)}=g_{1}\left(t_{m}\right)$ with $g_{1}(t)$ from (13a), whereas $g_{2}^{(m)}$ is given by (cf. (13b))

$$
g_{2}^{(m)}=\sum_{\ell=0}^{m-1}\left(\sum_{i=1}^{3}\left\|\mathbf{D}_{\Delta t}^{+} \mathbf{v}_{\mathrm{in}}^{(i)}\left(\cdot, t_{\ell}\right)\right\|_{\mathrm{H}_{00}^{5 / 2+\mu}\left(\Gamma_{\text {in }}^{(i)}\right)}^{2}+\sum_{i=1}^{2}\left\|\mathbf{D}_{\Delta t}^{+} \mathbf{v}_{\text {out }}^{(i)}\left(\cdot, t_{\ell}\right)\right\|_{\mathrm{H}_{00}^{5 / 2+\mu}\left(\Gamma_{\text {out }}^{(i)}\right)}^{2}+\left\|\mathbf{D}_{\Delta t}^{+} \mathbf{v}_{\mathrm{ac}}\left(\cdot, t_{\ell}\right)\right\|_{\mathrm{H}_{00}^{5 / 2+\mu}\left(\Gamma_{\mathrm{ac}}\right)}^{2}\right) \Delta t .
$$

\section{Theorem 3.1.}

Let $\left(\mathbf{v}_{h}^{(m)}, p_{h}^{(m)}, \mathrm{X}_{h}^{(m)}\right)_{m=0}^{M}$ be the solution of the semi-implicit Backward Euler/Forward Euler FE-IB (26a), (26b) and (27). In addition to the assumptions (8a) and (8b) let

$$
\max _{0 \leq m \leq M} g_{1}^{(m)} \leq \frac{\eta}{16 \rho C_{2}^{(1)} C_{\Omega}^{2}}
$$

be satisfied and suppose that the following CFL-condition holds true:

$$
\frac{\Delta t}{h} \leq \frac{\eta}{8 C_{\text {cell }}\left(\kappa_{e} \Lambda_{1}+\kappa_{b} \Lambda_{2}\right)}
$$

where the positive constants $C_{\Omega}, C_{\text {cell }}, C_{2}^{(1)}, \Lambda_{1}, \Lambda_{2}$ are from (15), (29), (30), and (31a). Then, there exists a positive constant $C$, depending on $\rho, \eta, \kappa_{e}, \kappa_{b}, C_{2}^{(k)}, 0 \leq k \leq 2$, and $C_{\Omega}, C_{\text {cell }}, \wedge_{1}, \wedge_{2}$ such that the following stability estimate is fulfilled:

$$
\begin{aligned}
\frac{\rho}{4}\left\|\mathbf{v}_{h}^{(m)}\right\|_{0, \Omega}^{2} & +\frac{\eta}{16} \sum_{\ell=0}^{m}\left\|\nabla \mathbf{v}_{h}^{(\ell)}\right\|_{0, \Omega}^{2} \Delta t+\frac{\kappa_{e}}{2}\left\|\frac{\partial \mathbf{X}_{h}^{(m)}}{\partial q}\right\|_{0,[0, L]}^{2}+\frac{\kappa_{b}}{2}\left\|\frac{\partial^{2} \mathbf{X}_{h}^{(m)}}{\partial q^{2}}\right\|_{0,[0, L]}^{2} \\
\leq C & \left(g_{1}^{(0)}\right)^{2}+\left(g_{1}^{(m)}\right)^{2}+\sum_{\ell=0}^{m-1}\left(\left(g_{1}^{(\ell)}\right)^{2}+\left(g_{2}^{(\ell)}\right)^{2}\right) \Delta t+\max _{0 \leq \ell \leq m-1} g_{1}^{(\ell)} \sum_{\ell=0}^{m}\left\|\mathbf{v}_{h}^{(\ell)}\right\|_{0, \Gamma}^{2} \Delta t \\
& \left.+\left\|\mathbf{v}_{h}^{(0)}\right\|_{0, \Omega}^{2}+\left\|\frac{\partial \mathbf{X}_{h}^{(0)}}{\partial q}\right\|_{0,[0, L]}^{2}+\left\|\frac{\partial^{2} \mathbf{X}_{h}^{(0)}}{\partial q^{2}}\right\|_{0,[0, L]}^{2}+\sum_{\ell=1}^{m-1}\left\|\frac{\partial \mathbf{X}_{h}^{(\ell)}}{\partial q}\right\|_{0,[0, L]}^{2} \Delta t+\sum_{\ell=1}^{m-1}\left\|\frac{\partial^{2} \mathbf{X}_{h}^{(\ell)}}{\partial q^{2}}\right\|_{0,[0, L]}^{2} \Delta t\right) .
\end{aligned}
$$


Proof. We choose $\mathbf{v}_{h}=\mathbf{v}_{h}^{(\ell+1)}-\widehat{\psi}_{h}^{(\ell+1)}$ in (26a), multiply the equation by $\Delta t$ and sum over $\ell$ from $\ell=0$ to $\ell=m-1$. We thus obtain

$$
\sum_{\ell=0}^{m-1}\left(\left(\rho \mathbf{D}_{\Delta t}^{+} \mathbf{v}_{h}^{(\ell)}, \mathbf{v}_{h}^{(\ell+1)}-\widehat{\psi}_{h}^{(\ell+1)}\right)_{0, \Omega}+a\left(\mathbf{v}_{h}^{(\ell+1)}, \mathbf{v}_{h}^{(\ell+1)}-\widehat{\psi}_{h}^{(\ell+1)}\right)\right) \Delta t=\sum_{\ell=0}^{m-1}\left\langle\mathbf{F}_{h}^{(\ell)}, \mathbf{v}_{h}^{(\ell+1)}-\widehat{\psi}_{h}^{(\ell+1)}\right\rangle_{h} \Delta t
$$

By partial summation we find

$$
\begin{aligned}
& \sum_{\ell=0}^{m-1}\left(\mathbf{D}_{\Delta t}^{+} \mathbf{v}_{h}^{(\ell)}, \mathbf{v}_{h}^{(\ell+1)}\right)_{0, \Omega} \Delta t=\frac{1}{2}\left(\left\|\mathbf{v}_{h}^{(m)}\right\|_{0, \Omega}^{2}-\left\|\mathbf{v}_{h}^{(0)}\right\|_{0, \Omega}^{2}\right)+\frac{1}{2} \Delta t \sum_{\ell=0}^{m-1}\left\|\mathbf{D}_{\Delta t}^{+} \mathbf{v}_{h}^{(\ell)}\right\|_{0, \Omega}^{2} \Delta t \\
& \sum_{\ell=0}^{m-1}\left(\mathbf{D}_{\Delta t}^{+} \mathbf{v}_{h}^{(\ell)}, \widehat{\psi}_{h}^{(\ell)}\right)_{0, \Omega} \Delta t=-\sum_{\ell=0}^{m-1}\left(\mathbf{v}_{h}^{(\ell)}, \mathbf{D}_{\Delta t}^{+} \widehat{\psi}_{h}^{(\ell+1)}\right)_{0, \Omega} \Delta t+\left(\mathbf{v}_{h}^{(m)}, \widehat{\psi}_{h}^{(m)}\right)_{0, \Omega}-\left(\mathbf{v}_{h}^{(0)}, \widehat{\psi}^{(0)}\right)_{0, \Omega} .
\end{aligned}
$$

Using (35a), (35b), Young's inequality with $\varepsilon_{1}>0$, and the Poincaré-Friedrichs inequality (15), the first term on the left-hand side in (34) can be bounded from below as follows:

$$
\begin{aligned}
\sum_{\ell=0}^{m-1}\left(\rho \mathbf{D}_{\Delta t}^{+} \mathbf{v}_{h}^{(\ell)}, \mathbf{v}_{h}^{(\ell+1)}-\widehat{\psi}_{h}^{(\ell+1)}\right)_{0, \Omega} \Delta t & \\
\geq & \frac{\rho}{4}\left\|\mathbf{v}_{h}^{(m)}\right\|_{0, \Omega}^{2}-\rho\left\|\mathbf{v}_{h}^{(0)}\right\|_{0, \Omega}^{2}-\rho\left\|\widehat{\boldsymbol{\psi}}_{h}^{(m)}\right\|_{0, \Omega}^{2}-\frac{\rho}{2}\left\|\widehat{\boldsymbol{\psi}}_{h}^{(0)}\right\|_{0, \Omega}^{2}-\varepsilon_{1} \rho \sum_{\ell=0}^{m-1}\left\|\mathbf{v}_{h}^{(\ell)}\right\|_{0, \Omega}^{2} \Delta t-\frac{\rho}{4 \varepsilon_{1}} \sum_{\ell=0}^{m-1}\left\|\mathbf{D}_{\Delta t}^{+} \widehat{\psi}_{h}^{(\ell)}\right\|_{0, \Omega}^{2} \Delta t \\
\geq & \frac{\rho}{4}\left\|\mathbf{v}_{h}^{(m)}\right\|_{0, \Omega}^{2}-\rho\left\|\mathbf{v}_{h}^{(0)}\right\|_{0, \Omega}^{2}-\rho\left\|\widehat{\psi}_{h}^{(m)}\right\|_{0, \Omega}^{2}-\frac{\rho}{2}\left\|\widehat{\psi}_{h}^{(0)}\right\|_{0, \Omega}^{2} \\
& \quad-\varepsilon_{1} \rho C_{\Omega}^{2}\left(\sum_{\ell=0}^{m-1}\left\|\nabla \mathbf{v}_{h}^{(\ell)}\right\|_{0, \Omega}^{2} \Delta t+\sum_{\ell=0}^{m-1}\left\|\mathbf{v}_{h}^{(\ell)}\right\|_{0, \Gamma}^{2} \Delta t\right)-\frac{\rho}{4 \varepsilon_{1}} \sum_{\ell=0}^{m-1}\left\|\mathbf{D}_{\Delta t}^{+} \widehat{\psi}_{h}^{(\ell)}\right\|_{0, \Omega}^{2} \Delta t .
\end{aligned}
$$

As in the proof of Theorem 2.3, for the second term on the left-hand side in (34) we deduce the following lower bound.

$$
\begin{aligned}
& \sum_{\ell=0}^{m-1} a\left(\mathbf{v}_{h}^{(\ell+1)}, \mathbf{v}_{h}^{(\ell+1)}-\widehat{\boldsymbol{\psi}}_{h}^{(\ell+1)}\right) \Delta t \\
& \quad \geq \eta \sum_{\ell=0}^{m-1}\left\|\nabla \mathbf{v}_{h}^{(\ell+1)}(\cdot, \tau)\right\|_{0, \Omega}^{2} \Delta t-\eta \sum_{\ell=0}^{m-1}\left\|\nabla \mathbf{v}_{h}^{(\ell+1)}\right\|_{0, \Omega}\left\|\nabla \widehat{\psi}_{h}^{(\ell+1)}\right\|_{0, \Omega} \Delta t-\rho \max _{0 \leq \ell \leq m} g_{1}\left(t_{\ell}\right) \sum_{\ell=0}^{m-1} \sum_{i, j=1}^{2}\left\|\mathbf{v}_{h, j}^{(\ell+1)}\right\|_{0, \Omega}\left\|\frac{\partial \mathbf{v}_{h, i}^{(\ell+1)}}{\partial x_{j}}\right\| \|_{0, \Omega} d \tau \\
& \quad \geq\left(\frac{\eta}{2}-\rho \sqrt{2} C_{\Omega}^{2} \max _{1 \leq \ell \leq m} g_{1}\left(t_{\ell}\right)\right) \sum_{\ell=0}^{m-1}\left\|\nabla \mathbf{v}_{h}^{(\ell+1)}\right\|_{0, \Omega}^{2} \Delta t-\frac{\rho \sqrt{2}}{2} C_{\Omega}^{2} \max _{1 \leq \ell \leq m} g_{1}\left(t_{\ell}\right) \sum_{\ell=0}^{m-1}\left\|\mathbf{v}_{h}^{(\ell+1)}\right\|_{0, \Gamma}^{2} \Delta t-\frac{\eta}{2} \sum_{\ell=0}^{m-1} g_{1}^{2}\left(t_{\ell}\right) \Delta t .
\end{aligned}
$$

Observing (25) and (27), for the right-hand side in (34) we obtain

$$
\begin{aligned}
\sum_{\ell=0}^{m-1}\left\langle\mathbf{F}_{h}^{(\ell)}, \mathbf{v}_{h}^{(\ell+1)}-\widehat{\psi}_{h}^{(\ell+1)}\right\rangle_{h} \Delta t & =-\kappa_{e} \sum_{\ell=0}^{m-1} \int_{0}^{L} \frac{\partial \mathbf{X}_{h}^{(\ell)}}{\partial q} \cdot \mathbf{D}_{\Delta t}^{+} \frac{\partial \mathbf{X}_{h}^{(\ell)}}{\partial q} d q \cdot \Delta t+\kappa_{b} \sum_{\ell=0}^{m-1} \sum_{i=1}^{M} \int_{q_{i-1}}^{q_{i}} \frac{\partial^{3} \mathbf{X}_{h}^{(\ell)}}{\partial q^{3}} \cdot \mathbf{D}_{\Delta t}^{+} \frac{\partial \mathbf{X}_{h}^{(\ell)}}{\partial q} d q \cdot \Delta t \\
& +\kappa_{e} \sum_{\ell=0}^{m-1} \int_{0}^{L} \frac{\partial \mathbf{X}_{h}^{(\ell)}}{\partial q} \cdot \mathbf{D}^{1} \widehat{\psi}_{h}^{(\ell+1)} \frac{\partial \mathbf{X}_{h}^{(\ell)}}{\partial q} d q \cdot \Delta t+\kappa_{b} \sum_{\ell=0}^{m-1} \int_{0}^{L} \frac{\partial^{2} \mathbf{X}_{h}^{(\ell)}}{\partial q^{2}} \cdot \mathbf{D}^{1} \widehat{\psi}_{h}^{(\ell+1)} \frac{\partial^{2} \mathbf{X}_{h}^{(\ell)}}{\partial q^{2}} d q \cdot \Delta t \\
& +\kappa_{b} \sum_{\ell=0}^{m-1} \int_{0}^{L} \frac{\partial^{2} \mathbf{X}_{h}^{(\ell)}}{\partial q^{2}} \cdot \mathbf{D}^{2} \widehat{\psi}_{h}^{(\ell+1)}\left(\frac{\partial \mathbf{X}_{h}^{(\ell)}}{\partial q}, \frac{\partial \mathbf{X}_{h}^{(\ell)}}{\partial q}\right) d q \cdot \Delta t
\end{aligned}
$$


Partial summation yields

$$
-\kappa_{e} \sum_{\ell=0}^{m-1} \int_{0}^{L} \frac{\partial \mathbf{X}_{h}^{(\ell)}}{\partial q} \cdot \mathbf{D}_{\Delta t}^{+} \frac{\partial \mathbf{X}_{h}^{(\ell)}}{\partial q} d q \cdot \Delta t=\kappa_{e} \sum_{\ell=1}^{m} \int_{0}^{L} \mathbf{D}_{\Delta t}^{-} \frac{\partial \mathbf{X}_{h}^{(\ell)}}{\partial q} \cdot \frac{\partial \mathbf{X}_{h}^{(\ell)}}{\partial q} d q \cdot \Delta t+\kappa_{e}\left(\int_{0}^{L}\left|\frac{\partial \mathbf{X}_{h}^{(0)}}{\partial q}\right|^{2} d q-\int_{0}^{L}\left|\frac{\partial \mathbf{X}_{h}^{(m)}}{\partial q}\right|^{2} d q\right)
$$

For the first term on the right-hand side in (38) it follows that

$$
\kappa_{e} \sum_{\ell=1}^{m} \int_{0}^{L} \mathrm{D}_{\Delta t}^{-} \frac{\partial \mathbf{X}_{h}^{(\ell)}}{\partial q} \cdot \frac{\partial \mathbf{X}_{h}^{(\ell)}}{\partial q} d q \cdot \Delta t=\kappa_{e} \sum_{\ell=0}^{m-1} \int_{0}^{L} \frac{\partial \mathrm{X}_{h}^{(\ell)}}{\partial q} \cdot \mathbf{D}_{\Delta t}^{+} \frac{\partial \mathrm{X}_{h}^{(\ell)}}{\partial q} d q \cdot \Delta t+\kappa_{e} \Delta t \sum_{\ell=0}^{m-1} \int_{0}^{L}\left|\mathbf{D}_{\Delta t}^{+} \frac{\partial \mathrm{X}_{h}^{(\ell)}}{\partial q}\right|^{2} d q \cdot \Delta t
$$

Taking (27), (29), and (30) into account, for the last term on the right-hand side in (39) we find

$$
\begin{aligned}
\int_{0}^{L}\left|\mathbf{D}_{\Delta t}^{+} \frac{\partial \mathbf{X}_{h}^{(\ell)}}{\partial q}\right|^{2} d q=\int_{0}^{L}\left|\frac{\partial}{\partial q}\left(\mathbf{v}_{h}^{(\ell+1)}\left(\mathbf{X}_{h}^{(\ell)}\right)\right)\right|^{2} d q & \leq \int_{0}^{L}\left|\nabla \mathbf{v}_{h}^{(\ell+1)}\left(\mathbf{X}_{h}^{(\ell)}\right)\right|^{2}\left|\frac{\partial \mathbf{X}_{h}^{(\ell)}}{\partial q} \| \frac{\partial \mathbf{X}_{h}^{(\ell)}}{\partial q}\right| d q \\
& \leq \Lambda_{1}\left\|\nabla \mathbf{v}_{h}^{(\ell+1)}\right\|_{0, \partial B_{h, t_{\ell}}}^{2} \leq C_{\text {cell }} \wedge_{1} h^{-1}\left\|\nabla \mathbf{v}_{h}^{(\ell+1)}\right\|_{0, \Omega}^{2} .
\end{aligned}
$$

Combining (38), (39) and (40) results in

$$
-\kappa_{e} \sum_{\ell=0}^{m-1} \int_{0}^{L} \frac{\partial \mathrm{X}_{h}^{(\ell)}}{\partial q} \cdot \mathbf{D}_{\Delta t}^{+} \frac{\partial \mathrm{X}_{h}^{(\ell)}}{\partial q} d q \cdot \Delta t \leq \frac{\kappa_{e}}{2}\left(\left\|\frac{\partial \mathrm{X}_{h}^{(0)}}{\partial q}\right\|_{[0, L]}^{2}-\left\|\frac{\partial \mathrm{X}_{h}^{(m)}}{\partial q}\right\|_{[0, L]}^{2}\right)+\frac{\kappa_{e}}{2} C_{\text {cell }} \Lambda_{1} h^{-1} \Delta t \sum_{\ell=0}^{m-1}\left\|\nabla \mathbf{v}_{h}^{(\ell+1)}\right\|_{0, \Omega}^{2} \Delta t
$$

In much the same way we obtain

$\kappa_{b} \sum_{\ell=0}^{m-1} \sum_{r=1}^{R} \int_{q_{r-1}}^{q_{r}} \frac{\partial^{3} \mathbf{X}_{h}^{(\ell)}}{\partial q^{3}} \cdot \mathbf{D}_{\Delta t}^{+} \frac{\partial \mathbf{X}_{h}^{(\ell)}}{\partial q} d q \cdot \Delta t \leq \frac{\kappa_{b}}{2}\left(\left\|\frac{\partial^{2} \mathbf{X}_{h}^{(0)}}{\partial q^{2}}\right\|_{[0, L]}^{2}-\left\|\frac{\partial^{2} \mathbf{X}_{h}^{(m)}}{\partial q^{2}}\right\|_{[0, L]}^{2}\right)+\frac{\kappa_{b}}{2} C_{\text {cell }} \wedge_{2} h^{-1} \Delta t \sum_{\ell=0}^{m-1}\left\|\nabla \mathbf{v}_{h}^{(\ell+1)}\right\|_{0, \Omega}^{2} \Delta t$

Choosing $\varepsilon_{1}=\eta /\left(4 \rho C_{\Omega}^{2}\right)$ in (36), observing (32), using (33) in (41) and (42), and estimating the remaining terms on the right-hand side in (37) from above, as in the proof of Theorem 2.3, allows to conclude.

\section{Remark 3.2.}

The CFL-condition (33) for the semi-implicit scheme means a restriction of the time-step size $\Delta t$, in particular depending on the size and shape, the stiffness, and the deformability of the immersed membrane as reflected by the constants $C_{\text {cell }}, \kappa_{e}, \kappa_{b}, \Lambda_{1}, \Lambda_{2}$. We refer to [17] for a systematical study of this issue with regard to the motion of RBCs.

\section{Results of numerical simulations}

We present the results of numerical simulations for two scenarios related to the separation of RBC cells and melanoma cells in a separation channel by SAWACS. All computations have been performed under Linux featuring Intel $\left(\right.$ Core ${ }^{T M}$ i3-2100 CPU $3.10 \mathrm{GHz}$ and 7.7 GB RAM. According to the set-up of the experiments conducted in the lab of the first author, we have considered a separation channel

$$
\Omega=(0 \mu \mathrm{m}, 300 \mu \mathrm{m}) \times(0 \mu \mathrm{m}, 220 \mu \mathrm{m})
$$


with three inflow boundaries

$$
\Gamma_{\text {in }}^{(1)}=\{0\} \times(30 \mu \mathrm{m}, 80 \mu \mathrm{m}), \quad \Gamma_{\text {in }}^{(2)}=\{0\} \times(100 \mu \mathrm{m}, 130 \mu \mathrm{m}), \quad \Gamma_{\text {in }}^{(3)}=\{0\} \times(150 \mu \mathrm{m}, 200 \mu \mathrm{m}),
$$

two outflow boundaries

$$
\Gamma_{\text {out }}^{(1)}=\{300\} \times(20 \mu \mathrm{m}, 100, \mu \mathrm{m}), \quad \Gamma_{\text {out }}^{(2)}=\{300\} \times(120 \mu \mathrm{m}, 200 \mu \mathrm{m}),
$$

and a boundary

$$
\Gamma_{\mathrm{ac}}=(135 \mu \mathrm{m}, 165 \mu \mathrm{m}) \times\{0\},
$$

where the SAWs enter the separation channel. The density $\rho$ and the dynamic viscosity $\eta$ have been chosen as

$$
\rho=1.0 \cdot 10^{3} \mathrm{~kg} / \mathrm{m}^{3}, \quad \eta=6.0 \cdot 10^{-3} \mathrm{~Pa} \cdot \mathrm{s}
$$

both for the carrier fluid and the fluid enclosed by the membrane of the RBC and the melanoma cell. We have considered an RBC of diameter $7.5 \mu \mathrm{m}$, perimeter $L=19.8 \mu \mathrm{m}$ and moduli [28]

$$
\kappa_{e}=6.0 \cdot 10^{-6} \mathrm{~N} / \mathrm{m}, \quad \kappa_{b}=2.0 \cdot 10^{-19} \mathrm{Nm},
$$

whereas the melanoma cell has been modeled as a sphere of diameter $16 \mu \mathrm{m}$ and moduli [3]

$$
\kappa_{e}=2.8 \cdot 10^{-4} \mathrm{~N} / \mathrm{m}, \quad \kappa_{b}=1.2 \cdot 10^{-16} \mathrm{Nm}
$$

Although the melanoma cell is stiffer than the RBC (larger values of $\kappa_{e}, \kappa_{b}$ ), it is much larger and less deformable (smaller values of $C_{\text {cell }}$ and $\Lambda_{1}, \Lambda_{2}$ ). According to Remark 3.2, the semi-implicit scheme requires a much smaller time step size $\Delta t$ for the motion of the RBC than for the motion of the melanoma cell (see details for Scenarios I and II below).

\section{Scenario I}

The first scenario represents an experimental set-up without SAW actuation (i.e., $\mathbf{v}_{\mathrm{ac}}=\mathbf{0}$ on $\Sigma_{\mathrm{ac}}$ ) where an RBC enters the separation channel through the inlet $\Gamma_{\text {in }}^{(2)}$ and the inflow velocities $v_{\text {in }}^{(i)}$ on $\Sigma_{\text {in }}^{(i)}, 1 \leq i \leq 3$, are chosen according to

$$
\begin{array}{lll}
v_{\mathrm{in}}^{(1)}=\left(v_{\mathrm{in}, 1}^{(1)}, v_{\mathrm{in}, 2}^{(1)}\right)^{T}, & v_{\mathrm{in}, 1}^{(1)}=\widehat{v}_{\mathrm{in}}^{(1)} m_{\mathrm{in}}^{(1)}\left(x_{2}\right)\left(x_{2}-b_{1}^{(1)}\right)\left(b_{2}^{(1)}-x_{2}\right) \cos \alpha, & v_{\mathrm{in}, 2}^{(1)}=\widehat{v}_{\mathrm{in}}^{(1)} m_{\mathrm{in}}^{(1)}\left(x_{2}\right)\left(x_{2}-b_{1}^{(1)}\right)\left(b_{2}^{(1)}-x_{2}\right) \sin \alpha, \\
v_{\mathrm{in}}^{(2)}=\left(v_{\mathrm{in}, 1}^{(2)}, v_{\mathrm{in}, 2}^{(2)}\right)^{T}, & v_{\mathrm{in}, 1}^{(2)}=\widehat{v}_{\mathrm{in}}^{(2)} m_{\mathrm{in}}^{(2)}\left(x_{2}\right)\left(x_{2}-b_{1}^{(2)}\right)\left(b_{2}^{(2)}-x_{2}\right), & v_{\mathrm{in}, 2}^{(2)}=0, \\
v_{\mathrm{in}}^{(3)}=\left(v_{\mathrm{in}, 1}^{(3)}, v_{\mathrm{in}, 2}^{(3)}\right)^{T}, & v_{\mathrm{in}, 1}^{(3)}=\widehat{v}_{\mathrm{in}}^{(3)} m_{\mathrm{in}}^{(3)}\left(x_{2}\right)\left(x_{2}-b_{1}^{(3)}\right)\left(b_{2}^{(3)}-x_{2}\right) \cos \alpha, & v_{\mathrm{in}, 2}^{(3)}=-\widehat{v}_{\mathrm{in}}^{(3)} m_{\mathrm{in}}^{(3)}\left(x_{2}\right)\left(x_{2}-b_{1}^{(3)}\right)\left(b_{2}^{(3)}-x_{2}\right) \sin \alpha .
\end{array}
$$

Here, $b_{1}^{(1)}=30, b_{1}^{(2)}=80, b_{1}^{(2)}=100, b_{2}^{(2)}=130, b_{1}^{(3)}=150, b_{2}^{(3)}=200$, and $m_{\mathrm{in}}^{(i)}\left(x_{2}\right), 1 \leq i \leq 3$, are smooth cut-off functions satisfying $m_{\text {in }}^{(i)}\left(x_{2}\right)=1$ on $\left[b_{1}^{(i)}+\varepsilon, b_{2}^{(i)}-\varepsilon\right]$ and vanishing at $b_{1}^{(i)}$ and $b_{2}^{(i)}$. The inflow velocities $\widehat{v}_{\text {in }}^{(i)}, 1 \leq i \leq 3$, and the angle $\alpha$ have been chosen by means of

$$
\widehat{v}_{\mathrm{in}}^{(1)}=5.0 \cdot 10^{-2} \mathrm{~m} / \mathrm{s}, \quad \widehat{v}_{\mathrm{in}}^{(2)}=1.0 \cdot 10^{-2} \mathrm{~m} / \mathrm{s}, \quad \widehat{v}_{\mathrm{in}}^{(3)}=10.0 \cdot 10^{-2} \mathrm{~m} / \mathrm{s}, \quad \alpha=\frac{\pi}{6} .
$$

The inflow velocities have been chosen according to the experiments carried out in the lab of the first author. In particular, the velocities are tuned so that without SAW actuation the default outlet of a cell is the lower one at the right boundary of the channel, cf. Figure 1 and Figure 2.

The motion of the RBC has been simulated by the semi-implicit scheme. The simulation covered the time period from the time instant where the RBC entered the separation channel to the time instant where the RBC left the channel. For the discretization in space we have chosen a uniform simplicial triangulation of the computational domain $\Omega$ by right isosceles with $h=1 / 3$ and 54243 Degrees of Freedom (DOFs), 48146 DOFs for the velocity and 6097 DOFs for the pressure. The CFL condition (33) required a time step size $\Delta t=1 / 400$. Larger time step sizes led to numerical instabilities. The computational time for the whole simulation was 3 hours and 51 minutes.

The result of the numerical simulation is displayed in Figure 5 which shows the velocity field and the position of the cell when the RBC enters the separation channel (left) and shortly before it leaves the channel (right). As can be seen, the RBC leaves the separation channel through the designated outlet $\Gamma_{\text {out }}^{(1)}$. The complete path of the RBC (indicated in Figure 5 (right)) is in accordance with the experimental results obtained in the lab of the first author. 

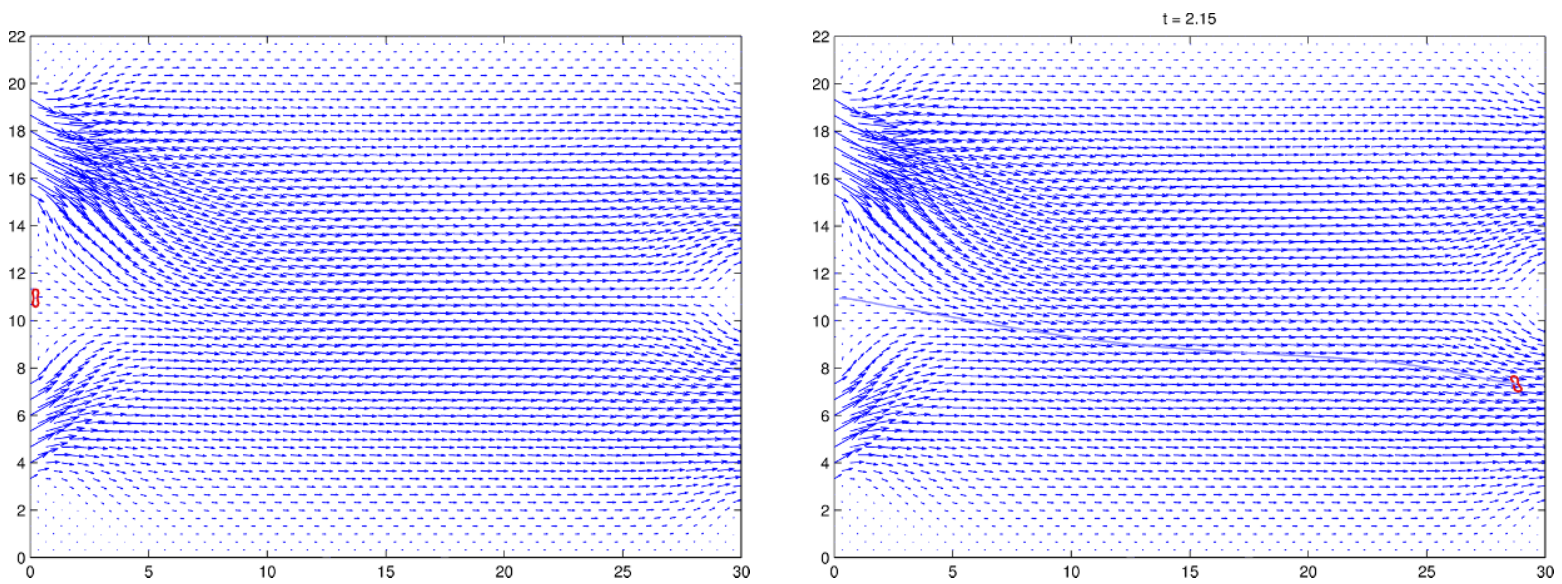

Figure 5. Velocity field and motion of an RBC without SAW actuation: Initial state after injection (left) and state shortly before the RBC leaves the separation channel (right).

\section{Scenario II}

The second scenario corresponds to a situation where a melanoma cell enters the separation channel through the inlet $\Gamma_{\text {in }}^{(2)}$, the inflow velocities are chosen as in Scenario I, and SAWs are created by an IDT with operating frequency $f=100.0 \mathrm{MHz}$ that enter the channel through $\Gamma_{\mathrm{ac}}$. The impact of the SAWs on the fluid flow is realized by an inflow velocity $v_{\mathrm{ac}}=\left(v_{\mathrm{ac}, 1}, v_{\mathrm{ac}, 2}\right), v_{\mathrm{ac}, 1}=0, v_{\mathrm{ac}, 2}=2 a \pi f m_{\mathrm{ac}}\left(x_{1}\right) \cos (2 \pi f t)$ on $\Sigma_{\mathrm{ac}}$. Here, $a=1.0 \cdot 10^{-9} \mathrm{~m}$ and $m_{\mathrm{ac}}\left(x_{1}\right)$ is a smooth cut-off function satisfying $m_{\mathrm{ac}}\left(x_{1}\right)=1$ on $\left[a_{1} / 2-d_{1}+\varepsilon, a_{1} / 2+d_{1}-\varepsilon\right]$ and vanishing at $a_{1} / 2-d_{1}$ and $a_{1} / 2+d_{1}$, cf. (3). The velocity $v_{a c}$ has been chosen according to the operational data of the IDT used in actual experiments.

The motion of the melanoma cell has been simulated by the semi-implicit scheme. As in Scenario I, the simulation covered the time period from the time instant where the cell entered the separation channel to the time instant where the cell left the channel. For the discretization in space we have chosen the same uniform simplicial triangulation with the same number of DOFs as in Scenario I. The CFL condition (33) only required a time step size $\Delta t=1 / 40$. The computational time for the whole simulation was 12 minutes.

Figure 6 displays the resulting velocity fields and the motion of the melanoma cell. Due to the SAW actuation, the path of the melanoma cell is diverted so that it leaves the separation channel through the outlet $\Gamma_{\text {out }}^{(2)}$. Again, the path of the cell is in accordance with experimental results obtained in the lab of the first author.
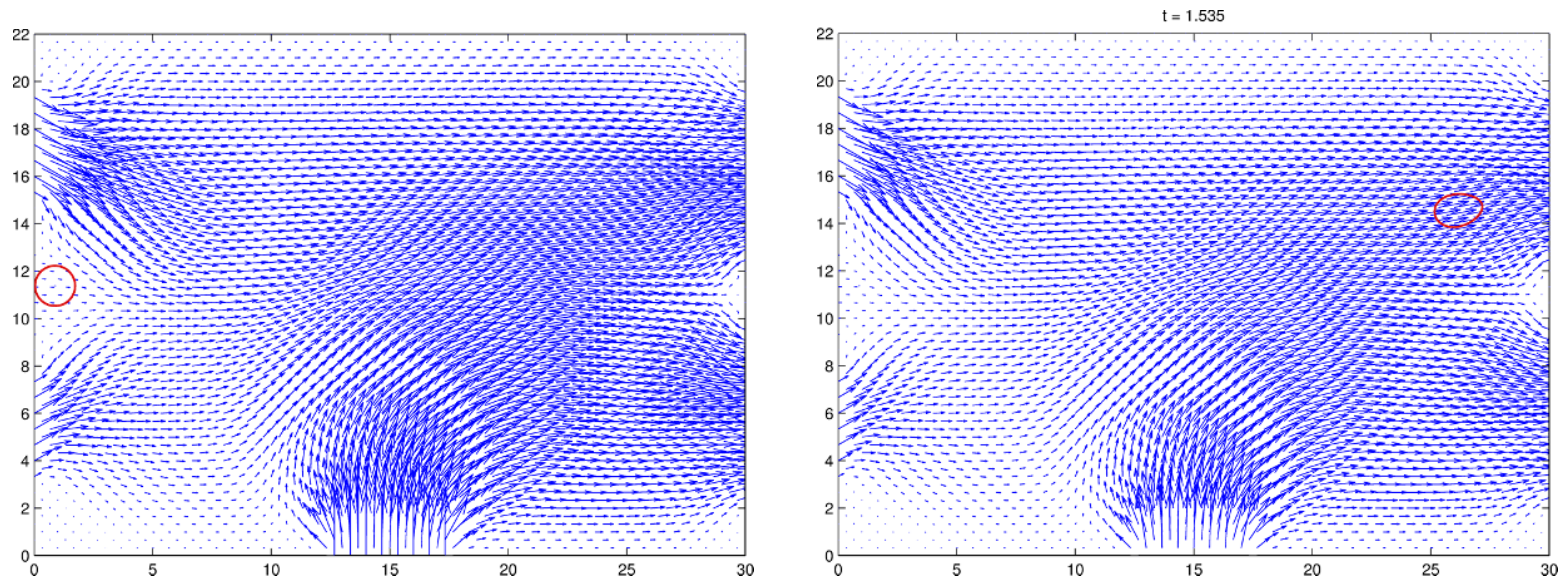

Figure 6. Velocity field and motion of a melanoma cell with SAW actuation: Initial state after injection (left) and state shortly before the cell leaves the separation channel (right). 


\section{Acknowledgements}

First author has been supported by the DFG Priority Program SPP 1506. Second author has been supported by the DFG Priority Programs SPP 1253 and SPP 1506, by the NSF grants DMS-0707602, DMS-0810176, DMS-0811153, DMS-0914788, DMS-1115658, and by the European Science Foundation within the Networking Programme 'OPTPDE'. Third author has been supported by the DFG Priority Program SPP 1253. Fourth author has been supported by the NSF grant DMS-1115658.

\section{References}

[1] Alberts B., Johnson A., Lewis J., Raff M., Roberts K., Walter P., Molecular Biology of the Cell, 4th ed., Garland Science, New York, 2002

[2] Antil H., Glowinski R., Hoppe R.H.W., Linsenmann C., Pan T.-W., Wixforth A., Modeling, simulation, and optimization of surface acoustic wave driven microfluidic biochips, J. Comput. Math., 2010, 28(2), 149-169

[3] Bekah D., Measurement of Viscoelastic Properties of Treated and Untreated Cancer Cells Using Passive Microrheology, MSc thesis, Ryerson University, Toronto, 2010, available at http://digitalcommons.ryerson.ca/dissertations/

[4] Boffi D., Cavallini N., Gastaldi L., Finite element approach to immersed boundary method with different fluid and solid densities, Math. Models Methods Appl. Sci., 2011, 21(12), 2523-2550

[5] Boffi D., Gastaldi L., A finite element approach for the immersed boundary method, Comput. \& Structures, 2003, 81(8-11), 491-501

[6] Boffi D., Gastaldi L., Heltai L., Numerical stability of the finite element immersed boundary method, Math. Models Methods Appl. Sci., 2007, 17(10), 1479-1505

[7] Brezzi F., Fortin M., Mixed and Hybrid Finite Element Methods, Springer Ser. Comput. Math., 15, Springer, BerlinHeidelberg-New York, 1991

[8] Carey J.L., McCoy J.P., Keren D.F. (Eds.), Flow Cytometry in Clinical Diagnostics, 4th ed., American Society for Clinical Pathology Press, Chicago, 2007

[9] Cui H.-H., Voldman J., He X.-F., Lim K.-M., Separation of particles by pulsed dielectrophoresis, Lab on a Chip, 2009, 9(16), 2306-2312

[10] Eisenstein M., Cell sorting: divide and conquer, Nature, 2006, 441, 1179-1185

[11] Eringen A.C., Maugin G.A., Electrodynamics of Continua I, Springer, Berlin-Heidelberg-New York, 1990

[12] Franke T., Braunmüller S., Frommelt T., Wixforth A., Sorting of solid and soft objects in vortices driven by surface acoustic waves, SPIE Proceedings, 2009, 7365, \# 736500

[13] Franke T., Braunmüller S., Schmid L., Wixforth A., Weitz D.A., Surface acoustic wave actuated cell sorting (SAWACS), Lab on a Chip, 2010, 10(6), 789-794

[14] Franke T., Hoppe R.H.W., Linsenmann C., Schmid L., Willbold C., Wixforth A., Numerical simulation of the motion of red blood cells and vesicles in microfluidic flows, Comput. Vis. Sci., 2011, 14(4), 167-180

[15] Gantner A., Hoppe R.H.W., Köster D., Siebert K.G., Wixforth A., Numerical simulation of piezoelectrically agitated surface acoustic waves on microfluidic biochips, Comput. Vis. Sci., 2007, 10(3), 145-161

[16] Hawley T.S., Hawley R.G. (Eds.), Flow Cytometry Protocols, 2nd ed., Methods in Molecular Biology, 263, Humana Press, Totowa, 2004

[17] Hoppe R.H.W., Linsenmann C., An adaptive Newton continuation strategy for the fully implicit finite element immersed boundary method, J. Comput. Phys., 2012, 231(14), 4676-4693

[18] Maugin G.A., Continuum Mechanics of Electromagnetic Solids, North-Holland Ser. Appl. Math. Mech., 33, NorthHolland, Amsterdam, 1988

[19] Pamme N., Continuous flow separations in microfluidic devices, Lab on a Chip, 2007, 7(12), 1644-1659

[20] Peskin C.S., Numerical analysis of flood flow in the heart, J. Comput. Phys., 1977, 25(3), 220-252

[21] Peskin C.S., The immersed boundary method, Acta Numer., 2002, 11, 479-517

[22] Petersson F., Åberg L., Swärd-Nilsson A.-M., Laurell T., Free flow acoustophoresis: Microfluidic-based mode of particle and cell separation, Analytical Chemistry, 2007, 79(14), 5117-5123 
[23] Qu B.-Y., Wu Z.-Y., Fang F., Bai Z.-M., Yang D.-Z., Xu S.-K., A glass microfluidic chip for continuous blood cell sorting by a magnetic gradient without labeling, Analytical and Bioanalytical Chemistry, 2008, 392(7-8), 1317-1324

[24] Seo J., Lean M.H., Kole A., Membrane-free microfiltration by asymmetrical inertial migration, Applied Physics Letters, 2007, 91(3), \# 033901

[25] Shapiro H.M., Practical Flow Cytometry, John Wiley \& Sons, Hoboken, 2003

[26] Shi J., Huang H., Stratton Z., Huang Y., Huang T.J., Continuous particle separation in a microfluidic channel via standing surface acoustic waves (SSAW), Lab on a Chip, 2009, 9(23), 3354-3359

[27] Shi J., Mao X., Ahmed D., Colletti A., Huang T.J., Focusing microparticles in a microfluidic channel with standing surface acoustic waves (SSAW), Lab on a Chip, 2008, 8(2), 221 ،-223

[28] Skalak R., Chien S., Handbook of Bioengineering, McGraw-Hill, New York, 1987

[29] Sklar L.A. (Ed.), Flow Cytometry for Biotechnology, Oxford University Press, New York, 2005

[30] Tartar L., An Introduction to Sobolev Spaces and Interpolation Spaces, Lect. Notes Unione Mat. Ital., 3, Springer, Berlin, 2007

[31] Valero A., Braschler T., Demierre N., Renaud P., A miniaturized continuous dielectrophoretic cell sorter and its applications, Biomicrofluidics, 2010, 4(2), \# 022807

[32] Zborowski M., Chalmers J.J., Magnetic cell sorting, In: Immunochemical Protocols, Methods in Molecular Biology, 295, Humana Press, New York, 2005, 291-300

[33] Zhu J., Xuan X., Curvature-induced dielectrophoresis for continuous separation of particles by charge in spiral microchannels, Biomicrofluidics, 2011, 5(2), \# 024111 